This item was submitted to Loughborough's Research Repository by the author.

Items in Figshare are protected by copyright, with all rights reserved, unless otherwise indicated.

\title{
A strategic and global manufacturing capacity management optimisation model: A Scenario-based multi-stage stochastic programming approach
}

\section{PLEASE CITE THE PUBLISHED VERSION}

https://doi.org/10.1016/j.omega.2019.01.004

\section{PUBLISHER}

(C) Elsevier

VERSION

AM (Accepted Manuscript)

\section{PUBLISHER STATEMENT}

This paper was accepted for publication in the journal Omega and the definitive published version is available at https://doi.org/10.1016/j.omega.2019.01.004.

\section{LICENCE}

CC BY-NC-ND 4.0

\section{REPOSITORY RECORD}

Sabet, Ehsan, Baback Yazdani, Ramez Kian, and Kostas Galanakis. 2019. "A Strategic and Global Manufacturing Capacity Management Optimisation Model: A Scenario-based Multi-stage Stochastic Programming Approach". figshare. https://hdl.handle.net/2134/36839. 


\title{
A strategic and global manufacturing capacity management optimisation model: A Scenario-based multi-stage stochastic programming approach
}

\author{
Ehsan Sabet $^{1 *}$ Baback Yazdani ${ }^{2} \quad$ Ramez Kian $^{2} \quad$ Kostas Galanakis $^{2}$ \\ ${ }^{1}$ Wolfson School of Mechanical, Electrical and Manufacturing Engineering \\ Loughborough University, Leicestershire LE11 3TU, UK \\ ${ }^{2}$ Nottingham Business School, Nottingham Trent University, Nottingham NG1 4FQ, UK
}

August 29, 2018

\begin{abstract}
Large-scale multinational manufacturing firms often $r$ equire a significant investment in production capacity and extensive management efforts in strategic planning in an uncertain business environment. In this research we first discuss $w$ hat d ecision terms and boundary conditions a holistic capacity management model for the manufacturing industry must contain. To better understand how these decision terms and constraints have been employed by the recent model developers in the area of capacity and resource management modelling for manufacturing, 69 optimisation-based (deterministic and stochastic) models have been carefully selected from 2000 to 2018 for a brief comparative analysis. The results of this comparison show although applying uncertainty into capacity modelling (in stochastic form) has received a greater deal of attention most recently (since 2010), the existing stochastic models are yet very simplistic, and not all the strategic terms have been employed in the current model developments in the field. This lack of a holistic approach although is evident in deterministic models too, the existing stochastic counterparts proved to include much less decision terms and inclusive constraints, which limits them to limited applications and may cause sub-optimal solutions. Employing this set of holistic decision terms and boundary conditions, this work develops a scenario-based multi-stage stochastic capacity management model, which is capable of modelling different strategic terms such as capacity level management (slight, medium and large capacity volume adjustment to increase/decrease capacity), location/relocation decisions, merge/decomposition options, and product management (R\&D, new product launch, product-to-plant and product-tomarket allocation, and product phase-out management). Possibility matrix, production rates, different financial terms and international taxes, inflation rates, machinery depreciation, investment lead-time and product cycle-time are also embedded in the model in order to make it more practical, realistic and sensitive to strategic decisions and scenarios. A step-by-step open-box validation has been followed while designing the model and a holistic black-box validation plan has been designed and employed to widely validate the model. The model then has been verified by deploying a real-scaled case of Toyota Motors UK (TMUK) decision of mothballing one of their production lines in the UK after the global recession in 2010.
\end{abstract}

Keywords: Stochastic programming, Optimisation modelling, Capacity Management, Manufacturing

\footnotetext{
${ }^{*}$ Corresponding author. Tel.: +44 1509227192.

E-mail addresses: e.sabet@lboro.ac.ac.uk (E. Sabet), baback.yazdani@ntu.ac.uk (B. Yazdani), ramez.kian@ntu.ac.uk (R. Kian), kostas.galanakis@ntu.ac.uk (K. Galanakis)
} 


\section{Introduction}

Resource management is one of the most important management tasks in manufacturing (Julka et al. 2007), and production capacity is the most strategic internal capability that manufacturing firms must create, sustain and plan for (Chen et al. 2002). Capacity management aims to ensure that a manufacturer has the 'right' capacity to act within a complex structure (Ambrosi 2010); and how best to 'utilise' their internal capabilities (Olhager et al. 2001). Due to the inherently parametric nature of the capacity management decisions, a quantitative approach has been more employed in this field (Julka 2008; Pidd 2003). However, an analytical decision-making model at best will only assist managers to better analyse and understand the trade-offs among available strategic choices (Eppen et al. 1989); and hence implementing a holistic set of important variables and decision terms in such models can improve the understanding of the trade-offs and prevent sub-optimal decisions, which is aimed by this work.

\subsection{Decision Terms and Constraints of a Holistic Strategic Capacity Management Model}

This section discusses the terms that must be embedded in a strategic capacity management model, which are categorised in four main groups of capacity level management, capacity location management, product and process management, and other terms as financial, political and environmental (Sabet 2012). Using 'input, control, output and mechanism' (ICOM) framework (Matta et al. 2005), the following sub-sections aim to introduce these critical decisions, and step by step configure the overall ICOM framework as summarised in Figure 1, which sets the boundary conditions for the model developed by this paper (Section 3).

\subsubsection{Capacity Level Management}

Adjusting production level to the long-term changes in demand, with minimum cost and lead-time implications, is the baseline for all capacity management models (Kauder and Meyr 2009). In manufacturing industry production capacities often change in bulk (so called 'lumpy nature' of the production capacity), indicating that the capacity changes in discontinuous and non-linear volumes (Olhager et al. 2001). Besides, manufacturing capacity often can change in 3 ranges of slight, medium and significant (Lin et al. 2010), in sequential form of overutilization, capacity expansion and new plant/line establishment for capacity increase, and underutilisation, mothball and capacity shut-down for capacity decrease scenarios. See Table 1. 
Table 1: ICOM summary for capacity level management- No term for mechanism

\begin{tabular}{|c|c|c|c|}
\hline & Output & Input & Control \\
\hline 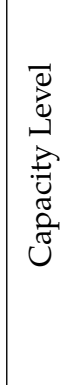 & $\begin{array}{l}\text { - Capacity increase (over- } \\
\text { utilization, expansion, new } \\
\text { plan) } \\
\text { - Capacity decrease (over- } \\
\text { utilization, mothball, shut- } \\
\text { down) }\end{array}$ & $\begin{array}{l}\text { - Maximum annual budget for invest- } \\
\text { ment } \\
\text { - Current capacities structure: location } \\
\text { (in relate to suppliers and customers), } \\
\text { operations costs, shut-down costs, } \\
\text { expansion costs, over and under- } \\
\text { utilisation costs, labour costs, etc. } \\
\text { - New plant choices and their cost } \\
\text { structure }\end{array}$ & $\begin{array}{l}\text { - Lumpy nature of capacity } \\
\text { change } \\
\text { - Investment lead-time } \\
\text { - Logical Constraints: non- } \\
\text { negativity, non-aticipativity, } \\
\text { non-simultaneity }\end{array}$ \\
\hline
\end{tabular}

\subsubsection{Capacity Location Management (Location, Relocation, Merge and Decomposition)}

In 1990's and 2000's, more than 75\% and 90\% of the biggest American companies invested in factories outside their countries respectively (Hamad and Fares Gualda 2008) and more recently an increasing number of EU and US companies are reshoring, which shows a need for the location/relocation decision terms in the capacity management models. Location decisions are functions of the geographical dispersion of the firm's suppliers, their manufacturing facilities, their sales regions and their investment portfolio (Kauder and Meyr 2009), as well as labour cost and energy costs in different locations, tariff and trade concessions, capital subsidies and logistics costs (Ferdows 1997). All these parametric terms can be employed in a holistic model. However, subjective factors, such as the company's policies, organisational learning through closeness to the customers and higher reliability and visibility to customers ought to be taken to the model as possible input options (MacCormack et al. 1994). The lead-time of the location/relocation decisions must also be considered in modelling to make it more realistic and sensitive to investment portfolios (Mula et al. 2006). Sometimes relocation decision is being made to centralise or decentralise the production capacities of a firm (by merging or decomposition), which can be embedded into a location/relocation model, using merge/decomposition possibility matrix. See Table 2 for the ICOM terms of location, relocation, merge and decomposition.

\subsubsection{Product and Process Management (product development and technology manage- ment)}

Product life-cycles are often much less than the capacity management planning horizon, and thus the entire product life-cycle curve, from the new product development to mass-production and final phase-out stage must be included in the time horizon of a capacity management 
Table 2: ICOM summary for Capacity location Management - No term for mechanism

\begin{tabular}{|c|c|c|c|}
\hline & Output & Input & Control \\
\hline 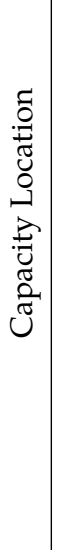 & $\begin{array}{l}\text { - Decision on different choices } \\
\text { of locations for a new plan. } \\
\text { - Decision on whether to close } \\
\text { a plant to open a new choice } \\
\text { of plant (relocation) } \\
\text { - Decision on whether to close } \\
\text { two or more current plant to } \\
\text { open a new choice of plant } \\
\text { (merge) } \\
\text { - Decision to close a current } \\
\text { plant and open two or more } \\
\text { new choice of new plants } \\
\text { (decomposition) }\end{array}$ & $\begin{array}{l}\text { - Maximum annual budget for invest- } \\
\text { ment } \\
\text { - Choices of new plants and current } \\
\text { plant structures (as detailed in Table } \\
\text { 1) } \\
\text { - Taxes, inflations, capital subsidies, } \\
\text { labour costs, energy costs } \\
\text { - Supply cost/tariff sensitivity to loca- } \\
\text { tions } \\
\text { - Sales sensitivity to locations (trans- } \\
\text { portation costs and tariffs) }\end{array}$ & $\begin{array}{l}\text { - Merge and decomposition } \\
\text { possibility matrix (what set } \\
\text { of current plant can be } \\
\text { merged for a new choice of } \\
\text { plant for merge case, and } \\
\text { what plant can be closed } \\
\text { down to open a set of } \\
\text { new plants for decomposi- } \\
\text { tion)Investment lead-time } \\
\text { - Investment lead-time } \\
\text { - Logical Constraints: non- } \\
\text { negativity, non-aticipativity, } \\
\text { non-simultaneity }\end{array}$ \\
\hline
\end{tabular}

model (Francas et al. 2009). The early phases which often called new product development (NPD) can be divided into two main phases of design or 'research and development' (R\&D) and new product launch (NPL). R\&D covers innovation, concept development and prototype making, and NPL is about launching a new product into plants, setups, first batch productions, and production ramp-ups. R\&D phase is often more centralised and carried out in research centres, while NPL phase requires some product-related investments in the allocated plants (Fleischmann et al. 2006). Besides, both R\&D and NPL phases are timely, and their investment lead-time must be considered in the product management models (Papageorgiou et al. 2001). Technology management in capacity and resource management models can be divided into two main domains of manufacturing flexibility, and facility replacement (technology obsolescence). Manufacturing flexibility is about the ability of a system to change its capacity quickly and economically (Ceryan and Koren 2009). It can be categorised into two types of 'product-mix' and 'volume flexibilities' (Kauder and Meyr 2009). Product-mix explains how a production facility can quickly and efficiently switch from one product to another. Volume flexibility explains how quickly and economically a production facility can adjust its production volume to chase the demand. 'Possibility matrix' and 'Production capacity rate' are being commonly used respectively, to model product-mix and volume flexibility in the capacity management models. Luss (1982) studies both physical depreciation and technology obsolescence in technology acquisition models. To formulate technology obsolescence modellers must simultaneously employ product life-cycles, product-plant investment requirements and the overhaul cost of facilities, all in a cost-based objective capacity management model ( $\mathrm{Wu}$ and Chuang 2010). Table 3 summarises ICOM terms for this section. 
Table 3: ICOM summary for product and process management - No term for mechanism

\begin{tabular}{|c|c|c|c|}
\hline & Output & Input & Control \\
\hline 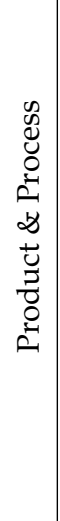 & $\begin{array}{l}\text { - Product to plant allocations } \\
\text { - Product to market allocation } \\
\text { - What choice of new prod- } \\
\text { ucts to develop, when and in } \\
\text { which plant } \\
\text { - Product re-launch decision } \\
\text { (when and in which plant) } \\
\text { - Choice of process technology } \\
\text { (in form of what choice of } \\
\text { new plant or expansion tech- } \\
\text { nology is selected, when val- } \\
\text { ume increase is needed) }\end{array}$ & $\begin{array}{l}\text { - Choice of process technology and } \\
\text { their cost structure and depreciation } \\
\text { - Choices of new plants and current } \\
\text { plant structures (as detailed in Table } \\
\text { 1) } \\
\text { - R\&D costs for each product family } \\
\text { - Product-related investment portfolio } \\
\text { in plants (for NPL) } \\
\text { - Current and new product life-cycles } \\
\text { (in sales forecast scenarios) }\end{array}$ & $\begin{array}{l}\text { - Product/plant possibility } \\
\text { matrix and product capacity } \\
\text { rate } \\
\text { - NPD and re-launch Invest- } \\
\text { ment lead-time }\end{array}$ \\
\hline
\end{tabular}

\subsubsection{Other Terms (Financial, Political and Environmental)}

Factors such as custom duties, inflation rates, tax on profit and value added tax have been mentioned as direct financial terms to be embedded in a strategic capacity management model (Fleischmann et al. 2006) to make a global capacity management model sensitive to location decisions (Verter and Dasci 2002). Although exchange rates directly affect capacity investment choices and relocation decisions (Farahani et al. 2010), there is no universally accepted exchange rate forecasting model (Bhutta et al. 2003). Besides, acquisition of the manufacturing resource is often of high lead-time; and therefore must be planned over a long term horizon (Olhager et al. 2001), for which uncertainty is an inevitable part (Chen et al. 2006). External sources of uncertainty, such as demand changes have been identified as the most disturbing and less controllable ones (Ahmed et al. 2003). However, many capacity planning models assume production should fulfil the entire demand, which may impose significant adjustments to the capacity level, even for a slight demand change. These unrealistic and sub-optimised solutions can be restrained by adapting an acceptable level of unsatisfied demand, which is usually associated with a penalty called 'unmet demand penalty' (Eppen et al. 1989). Although including supply chain selection decisions in capacity management models make them prohibitively complex, strategic capacity models can embed supply chain cost structure as a baseline for the supply chain (SC) related terms to make the models more sensitive to SC locations (Naraharisetti and Karimi 2010). Finally, although human resources and shift design may be considered as operational decisions, they affect some of the strategic ones such as capacity mothball and over/under utilisation; and thus, should not be entirely ignored in capacity management models. See Table 4 . 
Table 4: ICOM summary for financial and other terms - No term for mechanism

\begin{tabular}{|c|c|c|c|}
\hline & Output & Input & Control \\
\hline 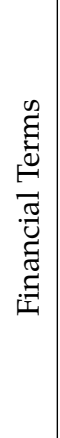 & $\begin{array}{l}\text { Shift management (in terms } \\
\text { of adding or removing a shift } \\
\text { to operations to change ca- } \\
\text { pacity level) }\end{array}$ & $\begin{array}{l}\text { - Custom duties (on supply and sales, } \\
\text { as a dependent of origin and destina- } \\
\text { tion countries) } \\
\text { - Cost of supply (as a dependent of sup- } \\
\text { plier location vs. manufacturing unit } \\
\text { locations) } \\
\text { - Inflation rates and tax in countries } \\
\text { where R\&D centres and manufactur- } \\
\text { ing plants and are located } \\
\text { - VAT in sales regions }\end{array}$ & $\begin{array}{l}\text { - Market uncertainty (sales } \\
\text { price and demand) } \\
\text { - Unmet demand penalties }\end{array}$ \\
\hline
\end{tabular}

Figure 1: The Model's Framework in an ICOM logic adapted from Matta et al. (2005)

\begin{tabular}{|c|c|c|c|c|c|}
\hline Input (Database) & \multicolumn{4}{|c|}{ Control (Constraints) } & Output (Results) \\
\hline \multirow{4}{*}{\begin{tabular}{|l|} 
Maximum annual budget for investment \\
Current manufacturing Structure: all \\
information about current plants including: \\
operations costs in detail, possibility and \\
cost of expansion, mothball, shutdown, etc.
\end{tabular}} & \multirow{3}{*}{\begin{tabular}{|c|} 
Market \\
Uncertainty \\
(Demand \\
volume and \\
Sales price)
\end{tabular}} & \multirow{5}{*}{\multicolumn{2}{|c|}{$\begin{array}{l}\text { Logical Constraints: } \\
\text { non-negativity, non- } \\
\text { anticipativity, non- } \\
\text { simultaneity, on-off } \\
\text { constraints and user- } \\
\text { defined logical } \\
\text { constraints }\end{array}$}} & \multirow{3}{*}{\begin{tabular}{|c|} 
Merge and \\
decomposition \\
possibility \\
matrix
\end{tabular}} & \multirow{2}{*}{$\begin{array}{c}\text { Investment Portfolio (when, } \\
\text { where and how much to } \\
\text { invest on capacities and } \\
\end{array}$} \\
\hline & & & & & \\
\hline & & & & & Product-to-Plant allocation \\
\hline & \multirow{3}{*}{\begin{tabular}{|c|} 
Product/plant \\
Possibility \\
matrix \& \\
capacity
\end{tabular}} & & & Lead-times & Product-to-Market plamning \\
\hline \multirow{2}{*}{$\begin{array}{l}\text { Alternative choices of future plants: } \\
\text { investment portfolio as well as operations } \\
\text { cost and other running costs }\end{array}$} & & & & $\begin{array}{l}\text { Unmet } \\
\text { demand }\end{array}$ & \\
\hline & & \multicolumn{2}{|c|}{$\begin{array}{l}\text { Lumpy nature of } \\
\text { capacity }\end{array}$} & penalty & Merge / Decomposition \\
\hline $\begin{array}{l}\text { Current and future Product Families and } \\
\text { their operations and manufacturing }\end{array}$ & \multirow{2}{*}{\multicolumn{4}{|c|}{ Net Present Value Maximisation }} & Capacity Volume \\
\hline Current and future market regions & & & & & mpngoment \\
\hline \begin{tabular}{|l|} 
Future Market: Stochastic demand and sales \\
price in different regions
\end{tabular} & \multirow{2}{*}{\multicolumn{2}{|c|}{\begin{tabular}{|c|} 
Scenario-based stochastic \\
modelling (see \\
methodology section) \\
\end{tabular}}} & \multirow{2}{*}{\multicolumn{2}{|c|}{$\begin{array}{l}\text { Programming, solution } \\
\text { and verification (see } \\
\text { methodology section) }\end{array}$}} & $\begin{array}{l}\text { New product management } \\
\text { and planning }\end{array}$ \\
\hline Logistics and supply and costs & & & & & \\
\hline
\end{tabular}

\subsubsection{ICOM Summary for a Holistic Strategic Capacity Management Model}

Figure 1 illustrates the boundary conditions of a holistic strategic capacity management model by summarising section 1.1 and Tables $1-4$, which are employed in the model development in Section 3.

\subsection{Recent Developments and Gaps in Strategic Capacity Optimisation Modelling}

To better understand how the recent capacity optimisation models have covered the terms and parameters discussed before as the essential terms for a holistic capacity management model, and to understand how the model proposed in this paper contributes to the knowledge and practices in the field of capacity management modelling, Tables 14 and 15 (see Appendix) compare the terms employed in the most recent 69 models in the field. These models were found and selected in an extensive search for the optimisation-based models from those that 
embedded at least one of the strategic objective terms that was discussed in the last section. Scholar search engines such as Googlescholar, ScienceDirect, Emarald, IEEE Xplore, JSTOR and Springer were exploited in this search and after initial filtering to most relevant papers to the topic of this research and those published recently (2000 to 2018) these 69 papers were selected for a comparative analysis. To remark academic gaps in alternative topics of manufacturing systems including quantity production, transfer and assembly lines, balancing and, sequencing, etc., the reader may refer to a recent survey in (Yang et al. 2018).

Table 14 shows although embedding uncertainty in the modelling practice has recently received significant attention in capacity modelling, still just under one-third of capacity management models are deterministic. However, comparing Tables 14 and 15, one can see deterministic models tend to embrace more terms and factors into the modelling than their stochastic counterparts, and thus yet remained more multi-angels. Figure 2, summarises the findings from Table 14, and shows how well these recent models manage to simultaneously embed different terms in their formulations and stay multi-purpose.

Figure 2: How recent models in capacity management have embedded strategic terms

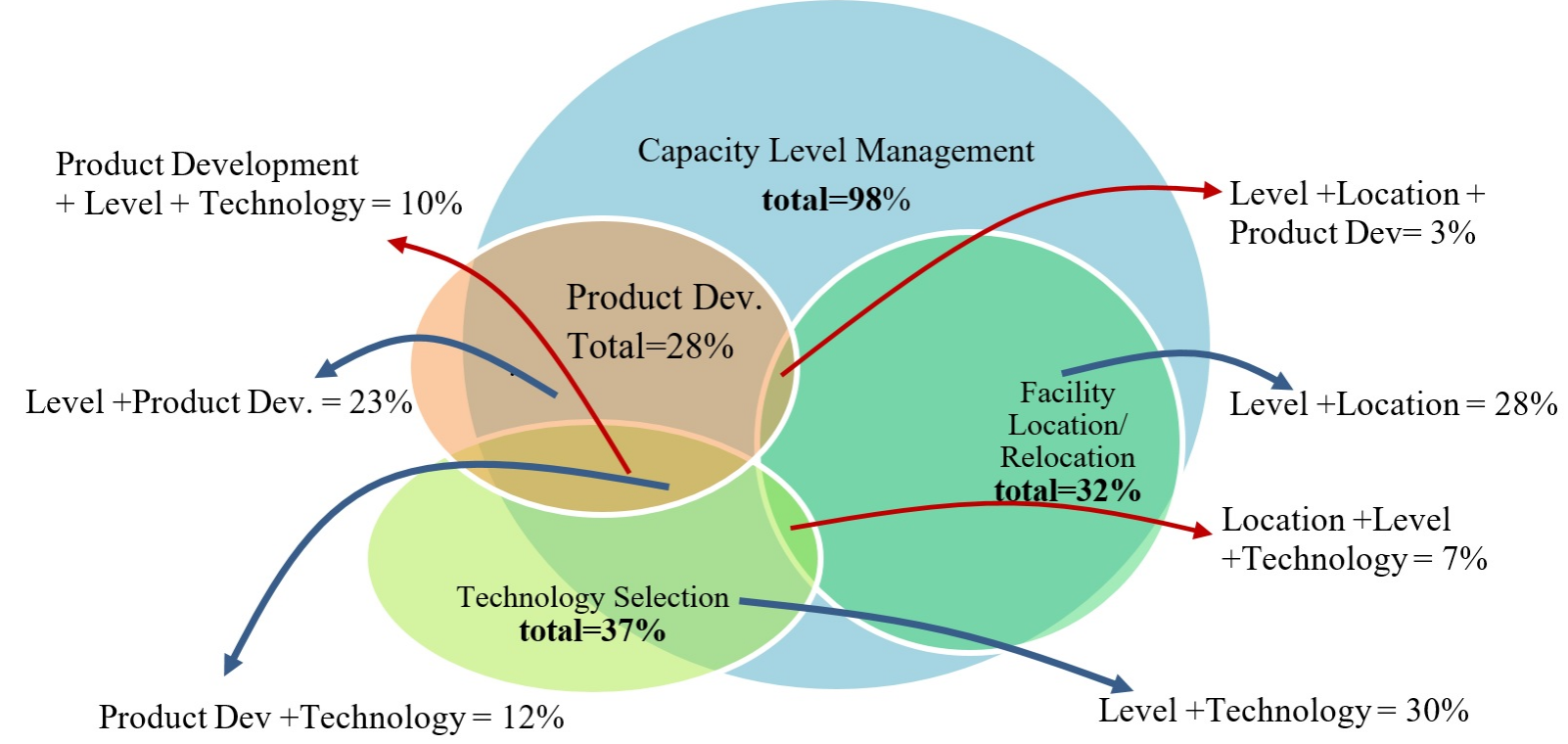

Although Table 14 shows capacity decrease modelling has widely left unattempted, Figure 2 illustrates still wide majority of the capacity management models have embedded at least one element of capacity level management in their models. However, only a few of these models have managed to embed at least three of the terms, and no model has yet covered all four strategic terms (Figure 2). Besides, as shown in Table 14, none of these models is capable of simultaneously managing all demand change possibilities (with over-utilisation, expansion and new capacity decisions in case of demand increase and under-utilisation, capacity mothball and 
capacity shutdown for demand decease scenarios), which limits the application of the models to a sub-set of potential applications. With regards to considering the product life-cycle in capacity management formulations, although the existing models have widely covered the mass production and decline phase, the early stages (concept design and product launch) have mainly left unattempted. Table 15 shows financial terms, detailed cost elements and control factors have been incompletely covered. As the result of this study, the model formulated in this paper (Section 3) aims to address these gaps and offer a more holistic capacity management model.

\section{Methodology}

Upon reviewing 103 models within the scope of resource planning under uncertainty, Peidro et al. (2009) categorised four quantitative approaches, namely analytical models, artificial intelligence-based models, simulation models and finally hybrid models. They summarise that the analytical approach has been more acknowledged and has had the fastest growth in resource management modelling (Peidro et al. 2009). As a part of analytical modelling, mathematical programming has been widely employed by peer scholars in strategic capacity management modelling (Hvolby and Steger-Jensen 2010; Melo et al. 2009; Mula et al. 2006). Traditionally in the real scale optimisation practices, often stochastic parameters have been replaced by their expected values (so called expected value problem) to make them deterministic and easier to programme and solve (Graves 2011). However, as explained in the last section, long-term capacity planning is subject to a vast uncertainty and thus simple estimations of expected values (or most probable scenarios) are no longer viable, and may lead to unrealistic capacity solutions (Barahona et al. 2005). Besides, statistical data are hardly reliable any more to forecast a long-term demand prospect, and thus a scenario-based stochastic programming has been employed as the most appropriate technique for log-term resource management modelling in Kauder and Meyr (2009), and therefore, is used in this paper, as well. The solution, validation and verification process are detailed later in this paper in Sections 4 and 5.

\section{Problem Statement and Model Formulation}

Led by ICOM framework in Section 1.1.5 (Figure 1), the rest of this section formulates the model and its constraints. The problem to be modelled is a long term strategic periodic capacity management problem by making decision on opening, closing, mothballing, reopening, expansion 
or overutilising different manufacturing plant in differently distributed geographical places under a stochastic demand or sales price in different regions by considering the transportation and cost of unmet demand, in such a way to maximise net present value of the firm. First we introduce the parameters and decision variables nomenclature in Table 5 and then describe different costs in the following subsections and subsequently, we present the objective function and mathematical model with the corresponding elaboration of the constraints.

\subsection{Fixed Investment Costs}

Some of the strategic capacity investments are long term (over one year time interval), and therefore, lead-time $(\eta)$ should be included in the programming. In this model the lead-time applies to the investments on new plants, expansions, new product developments and research and developments. If any of these investment decision variables $\left(Z_{. t .}^{\mathrm{New}}, Y_{. t .}^{\mathrm{Exp}}, Y_{. t .}^{\mathrm{NPL}}, Y_{. t .}^{\mathrm{RD}}\right)$ equals 1 at year $t$, investment starts a few years in advance (depending on the individual lead-time), so that the product or the production capacity is ready at the year $t$. Although to mothball a capacity the firm must also invest in redundancy and terminating supply contracts, it can often be conducted within the time intervals (one year) and need no lead-time. The same logic applies to shutdown decisions and reopening a mothballed capacity. Over-utilising a capacity requires annual investments for as long as the plant is over-utilised to invest in the extra times and third shifts, etc.. New product launch (NPL) occurs when a product is launched in any of the facilities for the first time. In such cases, a one-off launch cost is required for product-related investments (dedicated lines/machines, tooling, settings, training, first batch productions, system developments) and lead-time apply. However, relaunching an existing product after a long production-break needs a reset/changeover cost, but not as much as the NPL investment and it often needs no lead-time more than the time interval (one year). In a new product launch case, both $Y^{\mathrm{PL}}$ and $Y^{\mathrm{NPL}}$ equal 1; hence, the term $\left(Y^{\mathrm{PL}}-Y^{\mathrm{NPL}}\right)$ is applied to the extended objective function (2) for the product relaunch investments. 
Table 5: Nomenclatures list for the model formulation

\begin{tabular}{|c|c|c|c|c|c|}
\hline \multicolumn{4}{|c|}{ Parameters } & \multicolumn{2}{|c|}{ Variables } \\
\hline$\vartheta^{\mathrm{E}-\min }$ & $\begin{array}{l}\text { Min. capacity expansion rate } \\
\text { of plant } i \text {, out of nominal cap }\end{array}$ & $\Delta^{\mathrm{Opr}}$ & $\begin{array}{l}\text { Inflation rate on operations } \\
\text { cost }\end{array}$ & $X_{z t r j}^{\text {Unmet }}$ & $\begin{array}{l}\text { Amount of unmet product } j \\
\text { in region } r \text { in year } t \text { under } \\
\text { scenario } z\end{array}$ \\
\hline$\vartheta^{\mathrm{E}-\mathrm{max}}$ & $\begin{array}{l}\text { Max. capacity expansion rate } \\
\text { of plant } i, \text { out of nominal cap }\end{array}$ & $\Delta^{\operatorname{Inv}}$ & $\begin{array}{l}\text { Inflation rate on investment } \\
\text { cost }\end{array}$ & $X_{z t i j}^{\mathrm{A}}$ & $\begin{array}{l}\text { Amount of product } j \text { in plant } \\
i \text { in year } t, \text { under scenario } z\end{array}$ \\
\hline$\sigma_{i}^{\operatorname{Tax}}$ & $\begin{array}{l}\text { Profit tax rate in plant } i \text { loca- } \\
\text { tion }\end{array}$ & $\Delta^{\text {Sup }}$ & Inflation rate on supply cost & $X_{z t r i j}$ & $\begin{array}{l}\text { Amount of product } j \text { trans- } \\
\text { ported from plant } i \text { to region } \\
r \text { in year } t \text { under scenario } z\end{array}$ \\
\hline$\sigma_{r i}^{\text {Tariff }}$ & $\begin{array}{l}\text { Tariff rate of import from } \\
\text { plant } i \text { to region } r\end{array}$ & $\Delta^{\mathrm{D}}$ & $\begin{array}{l}\text { Inflation rate on transporta- } \\
\text { tion cost }\end{array}$ & $Y_{z t i j}^{\mathrm{A}}$ & $\begin{array}{l}\text { Binary variable which equals } \\
1 \text { if product } j \text { is produced in } \\
\text { plant } i \text { in year } t \text { under sce- } \\
\text { nario } z \text {; and } 0 \text {, otherwise }\end{array}$ \\
\hline$\sigma_{r}^{\mathrm{VAT}}$ & Value added tax in region $r$ & $\Delta^{\text {Unmet }}$ & $\begin{array}{l}\text { Inflation rate on unmet de- } \\
\text { mand penalty }\end{array}$ & $Y_{z t i}^{\mathrm{A}}$ & $\begin{array}{l}\text { Binary variable which equals } \\
1 \text { if plant } i \text { is is subject to } \\
\text { depreciation (either open or } \\
\text { mothballed) in year } t \text { under } \\
\text { scenario } z \text {; and } 0 \text {, otherwise }\end{array}$ \\
\hline$K_{i}^{\text {Initial }}$ & $\begin{array}{l}\text { Nominal capacity of plant } i \\
\text { before any volume change }\end{array}$ & $I_{i}^{\mathrm{New}}$ & $\begin{array}{l}\text { Capital investment to establish } \\
\text { new plant } i\end{array}$ & $Z_{z t i}^{\mathrm{New}}$ & $\begin{array}{l}\text { Binary variable which equals } \\
1 \text { if plant } i \text { is established in } \\
\text { year } t \text { under scenario } z \text {; and } \\
0 \text {, otherwise }\end{array}$ \\
\hline$\mu_{i}^{\mathrm{Max}}$ & $\begin{array}{l}\text { Normal capacity ratio (out of } \\
\text { maximum cap.) of plant } i\end{array}$ & $I_{i}^{\mathrm{Exp}}$ & $\begin{array}{l}\text { Capital investment to expand } \\
\text { plant } i\end{array}$ & $Y_{z t i}^{\operatorname{Exp}}$ & $\begin{array}{l}\text { Binary variable which equals } 1 \\
\text { if plant } i \text { is expanded in year } \\
t \text { under scenario } z \text {; and } 0, \\
\text { otherwise }\end{array}$ \\
\hline$\varepsilon_{i}^{\mathrm{Fr}}$ & $\begin{array}{l}\text { Redundancy rate on labour } \\
\text { cost, in case of plant } i \text { moth- } \\
\text { ball }\end{array}$ & $I_{i}^{\mathrm{Fr}}$ & $\begin{array}{l}\text { Capital investment to mothball } \\
\text { plant } i\end{array}$ & $Y_{z t i}^{\text {FrAll }}$ & $\begin{array}{l}\text { Binary variable which equals } \\
1 \text { if plant } i \text { has a mothballed } \\
\text { capacity in year } t \text { under sce- } \\
\text { nario } z \text {; and } 0 \text {, otherwise }\end{array}$ \\
\hline$\varepsilon_{i}^{\operatorname{Exp}}$ & $\begin{array}{l}\text { Increase rate on labour cost, in } \\
\text { case of plant } i \text { expansion }\end{array}$ & $I_{i}^{\mathrm{OprExp}}$ & $\begin{array}{l}\text { Extra annual operations cost } \\
\text { of plant } i \text {, if it has been ex- } \\
\text { panded }\end{array}$ & $Y_{z t i}^{\text {ExpAll }}$ & $\begin{array}{l}\text { Binary variable which equals } \\
1 \text { if plant } i \text { has ever been ex- } \\
\text { panded till year } t \text { under sce- } \\
\text { nario } z \text {; and } 0 \text { otherwise }\end{array}$ \\
\hline$\varepsilon_{i}^{\mathrm{Ou}}$ & $\begin{array}{l}\text { Increase rate on labour cost, in } \\
\text { case of overutilisation in plan } \\
i\end{array}$ & $I_{i}^{\mathrm{Ou}}$ & $\begin{array}{l}\text { Capital investment to } \\
\text { overutilise plant } i\end{array}$ & $Y_{z t i}^{\mathrm{Ou}}$ & $\begin{array}{l}\text { Binary variable which equals } \\
1 \text { if plant } i \text { is overutilised in } \\
\text { year } t \text { under scenario } z\end{array}$ \\
\hline$\eta_{j}^{\mathrm{RD}}$ & $\begin{array}{l}\text { Investment time-table to de- } \\
\text { sign the new product } j \text { (in } \\
\text { form of percentage over a few } \\
\text { time intervals) }\end{array}$ & $I_{j}^{\mathrm{RD}}$ & $\begin{array}{l}\text { Cost of designing product } j \text { in } \\
\text { research centre/headquarter }\end{array}$ & $Y_{z t j}^{\mathrm{RD}}$ & $\begin{array}{l}\text { Binary variable which equals } \\
1 \text { if product } j \text { is designed in } \\
\text { year } t \text { under scenario } z\end{array}$ \\
\hline$\eta_{i}^{\mathrm{New}}$ & $\begin{array}{l}\text { Investment timetable to es- } \\
\text { tablish plant } i \text { (in form of } \\
\text { percentage over a few time } \\
\text { intervals) }\end{array}$ & $I_{i}^{\mathrm{Re}}$ & $\begin{array}{l}\text { Capital investment to reopen } \\
\text { plant } i \text {, if it has been moth- } \\
\text { balled }\end{array}$ & $Y_{z t i}^{\operatorname{Re}}$ & $\begin{array}{l}\text { Binary variable which equals } \\
1 \text { if any capacity is reopened } \\
\text { in plant } i \text { in year } t \text { under sce- } \\
\text { nario } z\end{array}$ \\
\hline$\eta_{i}^{\text {Exp }}$ & $\begin{array}{l}\text { Investment timetable to ex- } \\
\text { pand plant } i \text { (in form of per- } \\
\text { centage over a few time inter- } \\
\text { vals) }\end{array}$ & $I_{i}^{\mathrm{OprFr}}$ & $\begin{array}{l}\text { Annual maintenance cost of } \\
\text { plant } i, \text { if it has been moth- } \\
\text { balled }\end{array}$ & $Y_{z t i}^{\mathrm{Fr}}$ & $\begin{array}{l}\text { Binary variable which equals } \\
1 \text { if plant } i \text { is mothballed in } \\
\text { year } t \text { under scenario } z \text {; and } \\
0, \text { otherwise }\end{array}$ \\
\hline$\eta_{i j}^{\mathrm{NPL}}$ & $\begin{array}{l}\text { Timetable of launching prod- } \\
\text { uct } j \text { in plant } i \text { for the first } \\
\text { time (in form of percentage } \\
\text { over a few time intervals) }\end{array}$ & $I_{i}^{\mathrm{NPL}}$ & $\begin{array}{l}\text { Cost of launching product } j \\
\text { in plant } i \text { for the first time }\end{array}$ & $Y_{z t i j}^{\mathrm{NPL}}$ & $\begin{array}{l}\text { Binary variable which equals } \\
1 \text { if NPL occurs for product } \\
j \text { in plant } i \text { in year } t \text { under } \\
\text { scenario } z\end{array}$ \\
\hline$l_{j}$ & $\begin{array}{l}\text { Maximum number of plants } \\
\text { to produce product } j\end{array}$ & $I_{i j}^{\mathrm{PL}}$ & $\begin{array}{l}\text { Cost of relaunching product } j \\
\text { in plant } i \text {, after a production } \\
\text { break }\end{array}$ & $Y_{z t i j}^{\mathrm{PL}}$ & $\begin{array}{l}\text { Binary variable which equals } \\
1 \text { if PL occurs for product } j \\
\text { in plant } i \text { in year } t \text { under sce- } \\
\text { nario } z\end{array}$ \\
\hline$n_{i}^{\operatorname{Max}}$ & $\begin{array}{l}\text { Maximum possible products to } \\
\text { be produced in plant } i\end{array}$ & $I_{i}^{\mathrm{W}}$ & $\begin{array}{l}\text { Annual work force cost of } \\
\text { plant } i\end{array}$ & $Y_{z t i}^{\mathrm{ExpW}}$ & $\begin{array}{l}\text { Binary variable which equals } 1 \\
\text { if in- } \text { use plant } i \text { has ever been }_{\text {expanded until year } t \text { under }} \\
\text { scenario } z\end{array}$ \\
\hline$d_{z t r j}$ & $\begin{array}{l}\text { Demand for product } j \text { in re- } \\
\text { gion } r \text { in year } t \text { under sce- } \\
\text { nario } z\end{array}$ & $I_{i}^{\mathrm{Opr}}$ & $\begin{array}{l}\text { Annual operations cost of } \\
\text { plant } i\end{array}$ & $Y_{z t i}^{\mathrm{ExpW}}$ & $\begin{array}{l}\text { Binary variable which takes } 1 \\
\text { if plant } i \text { is in use in year } t \\
\text { under scenario } z\end{array}$ \\
\hline$b_{t}$ & $\begin{array}{l}\text { Maximum investment budget } \\
\text { in year } t\end{array}$ & $I_{i}^{\mathrm{Cl}}$ & $\begin{array}{l}\text { Fixed cost of shutting down } \\
\text { plant } i\end{array}$ & $Y_{z t i}^{\mathrm{Cl}}$ & $\begin{array}{l}\text { Binary decision variable which } \\
\text { equals } 1 \text { if plant } i \text { is closed in } \\
\text { year } t \text { under scenario } z\end{array}$ \\
\hline$n_{i}^{\text {Merge }}$ & $\begin{array}{l}\text { How many plants should be } \\
\text { merged together to form plant } \\
i\end{array}$ & $C_{i j}^{\text {Sup }}$ & $\begin{array}{l}\text { Unit cost of supply for prod- } \\
\text { uct } j \text { in plant } i\end{array}$ & $K_{z t i}^{\mathrm{Max}}$ & $\begin{array}{l}\text { Nominal capacity of plant } i \text { in } \\
\text { year } t \text { under scenario } z\end{array}$ \\
\hline$M$ & A sufficiently large number & $C_{r i j}^{\mathrm{D}}$ & $\begin{array}{l}\text { Unit cost of transp. product } j \\
\text { from plant } i \text { to region } r\end{array}$ & $K_{z t i}^{\mathrm{Cl}}$ & $\begin{array}{l}\text { Shutdown capacity amount of } \\
\text { plant } i \text { in year } t \text { under sce- } \\
\text { nario } z\end{array}$ \\
\hline$P_{z}$ & Probability of scenario $z$ & $C_{i j}^{\text {Penalty }}$ & $\begin{array}{l}\text { Unit unmet demand penalty } \\
\text { for product } j \text { in region } r\end{array}$ & $K_{z t i}^{\text {FrAll }}$ & $\begin{array}{l}\text { Cumulative amount of moth- } \\
\text { balled capacity for plant } i \text { in } \\
\text { year } t \text { under scenario } z\end{array}$ \\
\hline$P_{i}^{\text {Merge }}$ & $\begin{array}{l}\text { The combination of the plants } \\
\text { that should be merged to form } \\
\text { plan } i\end{array}$ & $C_{i j}^{\mathrm{Unit}}$ & $\begin{array}{l}\text { Any other unit cost of produc- } \\
\text { ing product } j \text { in plant } i\end{array}$ & $K_{z t i}^{\mathrm{Fr}}$ & $\begin{array}{l}\text { Amount of capacity moth- } \\
\text { balled in plant } i \text { in year } t \text { un- } \\
\text { der scenario } z\end{array}$ \\
\hline$\gamma_{i j}$ & $\begin{array}{l}\text { Cap. volume rate of product } j \\
\text { in plant } i\end{array}$ & $C_{z t r j}^{\text {Sale }}$ & $\begin{array}{l}\text { Unit sales price of product } \\
j \text { in region } r \text { in year } t \text { and } \\
\text { scenario } z\end{array}$ & $K_{z t i}^{\operatorname{Exp}}$ & $\begin{array}{l}\text { Expanded capacity amount of } \\
\text { plant } i \text { in year } t \text { under sce- } \\
\text { nario } z\end{array}$ \\
\hline$E_{i}$ & $\begin{array}{l}\text { Maximum number of times } \\
\text { for possible expansion for } \\
\text { plant } i\end{array}$ & $\rho$ & Discount rate & $K_{z t i}^{\mathrm{Re}}$ & $\begin{array}{l}\text { Reopened capacity amount of } \\
\text { plant } i \text { in year } t \text { under sce- } \\
\text { nario } z\end{array}$ \\
\hline
\end{tabular}




\subsection{Variable/Operations Costs}

Transportation costs and unmet demand penalties are both unit-based costs. Unmet demand penalties should at least cover the net profit margin of the loss of sales, but it can also include the opportunity lost and brand damage (Eppen et al. 1989). However, penalties must have no tax-related implications. Annual workforce cost is a function of the plant location and can be different if a plant is normally utilised or overutilised, and if they are expanded or mothballed ( $\varepsilon$ is the proportion which adds to or cuts from the normal utilisation situation due to overutilisation and expansion, or mothball). Besides, to make the model sensitive to global capacity decisions and location/relocation decisions VAT and tariff taxes must be directly employed to the model as a function of location of the plants and sales regions. Other annual operations costs, maintenance costs and overheads also apply whenever a plant is functional (utilised or mothballed, but not closed). This model is not aimed to design the supply chain network. However, to avoid unrealistic simplification of ignoring the impact of supply chain network design on capacity location and planning, a location-sensitive supply cost has been applied to the model, as also supported by other researchers (Dal-Mas et al. 2011).

\subsection{Objective Function}

This model aims to maximise the net present value (NPV) under demand and sales price uncertainty (scenarios). A one-year time interval is set for the model, as cited for the strategic capacity planning by Fleischmann et al. (2006). A minimum of 10 years is suggested for capacity management models in manufacturing (Bhutta et al. 2003) and automotive industry (Kauder and Meyr 2009). Since R\&D activities in large and multi-national manufacturing firms often happen in their headquarters and research centres rather than their individual plants, $R \& D$ investments do not depend on the sales regions or plant locations. Thus the tax/incentive rates on these investments are not included in the objective function below,

$$
\max (N P V)=\max \sum_{z} P_{z} \sum_{t=1}^{T}\left\{(1+\rho)^{-t}\left[\sum_{i=1}\left(1-\sigma_{i}^{\mathrm{Tax}}\right)\left(\mathcal{R}_{z t i}-\mathcal{I}_{z t i}-\mathcal{O}_{z t i}\right)-\mathcal{D}_{z, t}\right]\right\}
$$

where $\mathcal{R}_{z t i}, \mathcal{I}_{z t i}$ and $\mathcal{O}_{z t i}$ respectively denote revenue, investment cost and operations cost for plant $i$ at year $t$ under scenario $z$ while $\mathcal{D}$ corresponds to R\&D cost.

Profit is the sum of sales in different regions over years under different demand and sales price scenarios minus the aforementioned costs. The objective function is extended in (2) to enable the decision-makers to test the impact of different inflation and tax scenarios on the 
location decisions, or different inflation rates $(\Delta)$ on investments, operations, supplies and distributions logistics as,

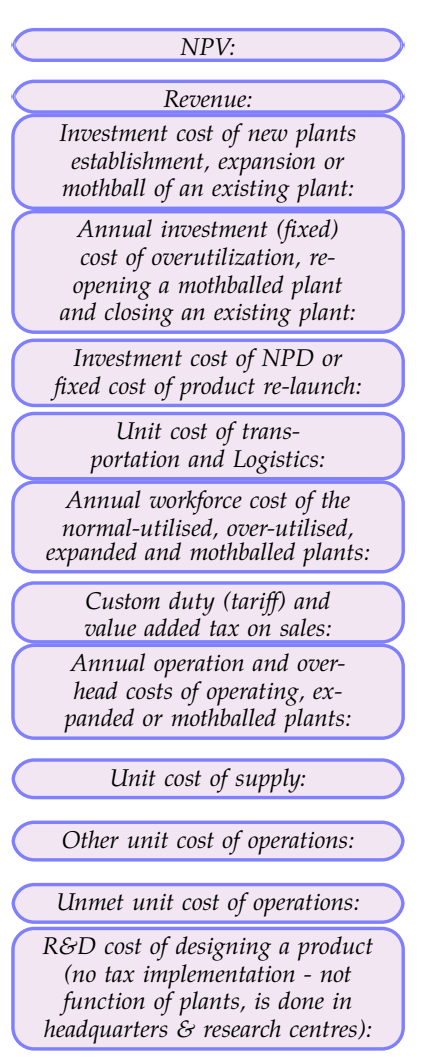

$$
\begin{aligned}
& \max \sum_{z} P_{z} \sum_{t=1}^{T}(1+\rho)^{-t}\{ \\
& +\sum_{r, i, j}\left(1-\sigma_{i}^{\text {Tax }}\right)\left(C_{z t r j}^{\text {Sale }} X_{z t r i j}\right) \\
& -\left[\sum_{i}\left(1-\sigma_{i}^{\mathrm{Tax}}\right)\left(1+\Delta^{\mathrm{Inv}}\right)^{t}\left(I_{i}^{\mathrm{New}} \eta_{i}^{\mathrm{New}} Z_{z t i}^{\mathrm{New}}+I_{i}^{\mathrm{Exp}} \eta_{i}^{\mathrm{Exp}} Y_{z t i}^{\mathrm{Exp}}+I_{i}^{\mathrm{Fr}} Y_{z t i}^{\mathrm{Fr}}\right)\right] \\
& -\left[\sum_{i}\left(1-\sigma_{i}^{\mathrm{Tax}}\right)\left(1+\Delta^{\mathrm{Inv}}\right)^{t}\left(I_{i}^{\mathrm{Ou}} Y_{z t i}^{\mathrm{Ou}}+I_{i}^{\mathrm{Re}} Y_{z t i}^{\mathrm{Re}}+I_{i}^{\mathrm{Cl}} Y_{z t i}^{\mathrm{Cl}}\right)\right] \\
& -\left[\sum_{i, j}\left(1-\sigma_{i}^{\mathrm{Tax}}\right)\left(I_{i}^{\mathrm{NPL}} \eta_{i j}^{\mathrm{NPL}} Y_{z t i j}^{\mathrm{NPL}}+I_{i}^{\mathrm{PL}}\left(Y_{z t i j}^{\mathrm{PL}}-Y_{z t i j}^{\mathrm{NPL}}\right)\right)\right] \\
& -\left[\sum_{r, i, j}\left(1-\sigma_{i}^{\mathrm{Tax}}\right)\left(1+\Delta^{\mathrm{D}}\right)^{t} C_{r i j}^{\mathrm{D}} X_{z t r i j}\right] \\
& -\left[\sum_{i}\left(1-\sigma_{i}^{\mathrm{Tax}}\right)\left(1+\Delta^{\mathrm{Opr}}\right)^{t} I_{i}^{\mathrm{W}}\left(Y_{z t i}^{\mathrm{A}}+\varepsilon_{i}^{\mathrm{Ou}} Y_{z t i}^{\mathrm{Ou}}+\varepsilon_{i}^{\mathrm{Ou}} Y_{z t i}^{\mathrm{ExpW}}-\varepsilon_{i}^{\mathrm{Fr}} Y_{z t i}^{\mathrm{FrAll}}\right)\right] \\
& -\left[\sum_{r, i, j}\left(1-\sigma_{i}^{\mathrm{Tax}}\right)\left(\sigma_{r i}^{\mathrm{Tariff}}+\sigma_{r}^{\mathrm{VAT}}\left(1+\sigma_{r i}^{\mathrm{Tariff}}\right)\right) C_{z r j}^{\mathrm{Sale}} X_{z t r i j}\right] \\
& -\left[\sum_{i}\left(1-\sigma_{i}^{\mathrm{Tax}}\right)\left(1+\Delta^{\mathrm{Opr}}\right)^{t}\left(I_{i}^{\mathrm{Opr}} Y_{z t i}^{\mathrm{ExpW}}+I_{i}^{\mathrm{OprExp}} Y_{z t i}^{\mathrm{ExpW}}+I_{i}^{\mathrm{OprFr}} Y_{z t i}^{\mathrm{FrAll}}\right)\right] \\
& -\left[\sum_{i, j}\left(1-\sigma_{i}^{\mathrm{Tax}}\right)\left(1+\Delta^{\mathrm{Sup}}\right)^{t} C_{i j}^{\mathrm{Sup}} X_{z t i j}^{\mathrm{A}}\right] \\
& \text { - }\left[\sum_{i, j}\left(1-\sigma_{i}^{\mathrm{Tax}}\right)\left(1+\Delta^{\mathrm{Opr}}\right)^{t} C_{i j}^{\mathrm{Unit}} X_{z t i j}^{\mathrm{A}}\right] \\
& -\left[\sum_{i, r}\left(1+\Delta^{\text {Unmet }}\right)^{t} C_{i j}^{\text {Penalty }} X_{z t r j}^{\text {Unmet }}\right] \\
& -\left[\sum_{j}\left(1+\Delta^{\mathrm{Inv}}\right)^{t} I_{j}^{\mathrm{RD}} \eta_{j}^{\mathrm{RD}} Y_{z t j}^{\mathrm{RD}}\right] \\
& \} \text {. }
\end{aligned}
$$

\subsection{Mathematical Model}

\section{$\max \quad N P V$}

s.t.

$$
\begin{array}{ll}
K_{z t i}^{\mathrm{Max}}=K_{z, t-1, i}^{\mathrm{Max}}+Z_{z, t-1, i}^{\mathrm{New}} K_{i}^{\mathrm{Initial}}+K_{z, t-1, i}^{\mathrm{Exp}}-K_{z, t-1, i}^{\mathrm{Fr}}-K_{z, t-1, i}^{\mathrm{Cl}}+K_{z, t-1, i}^{\mathrm{Re}} & \forall z, t, i \\
Y_{z t i}^{\mathrm{Exp}} \leq K_{z t i}^{\mathrm{Exp}} \leq M Y_{z t i}^{\mathrm{Exp}} & \forall i, t, z \\
Y_{z t i}^{\mathrm{Fr}} \leq K_{z t i}^{\mathrm{Fr}} \leq M Y_{z t i}^{\mathrm{Fr}} & \forall i, t, z \\
Y_{z t i}^{\mathrm{Re}} \leq K_{z t i}^{\mathrm{Re}} \leq M Y_{z t i}^{\mathrm{Re}} & \forall i, t, z \\
Y_{z t i}^{\mathrm{Cl}} \leq K_{z t i}^{\mathrm{Cl}} \leq M Y_{z t i}^{\mathrm{Cl}} & \forall i, t, z \\
\sum_{j} X_{z t i j}^{\mathrm{A}} / \gamma_{i j} \leq K_{z t i}^{\mathrm{Max}} & \forall i, t, z \\
\sum_{j}\left(X_{z t i j}^{\mathrm{A}} / \gamma_{i j}\right)-M Y_{z t i}^{\mathrm{Ou}} \leq \mu_{i}^{\mathrm{Max}} K_{z t i}^{\mathrm{Max}} & \forall z, t, i \\
\sum_{j}\left(X_{z t i j}^{\mathrm{A}} / \gamma_{i j}\right)+M\left(1-Y_{z t i}^{\mathrm{Ou}} \geq \mu_{i}^{\mathrm{Max}} K_{z t i}^{\mathrm{Max}}\right. &
\end{array}
$$




$$
\begin{aligned}
& \sum_{t=1}^{T} Z_{z t i}^{\mathrm{New}} \leq 1 \\
& \sum_{t=1}^{T} Y_{z t i}^{\operatorname{Exp}} \leq E_{i} \\
& K_{z t i}^{\text {Exp }} \leq Y_{z t i}^{\text {Exp }} \vartheta^{\text {E-max }} K_{i}^{\text {Initial }} \\
& Y_{z t i}^{\text {Exp }} \vartheta^{\text {E-min }} K_{i}^{\text {Initial }} \leq K_{z t i}^{\text {Exp }} \\
& K_{z t i}^{\mathrm{Fr}}+M\left(1-Y_{z t i}^{\mathrm{Fr}}\right) \geq K_{z t i}^{\mathrm{Max}} \\
& K_{z t i}^{\mathrm{Fr}} \leq K_{z t i}^{\mathrm{Max}} \\
& K_{z t i}^{\mathrm{FrAll}}=K_{z, t-1, i}^{\mathrm{FrAll}}+\left(K_{z, t-1, i}^{\mathrm{Fr}}-K_{z, t-1, i}^{\mathrm{Re}}\right) \\
& K_{z t i}^{\mathrm{Re}}+M\left(1-Y_{z t i}^{\mathrm{Re}}\right) \geq K_{z t i}^{\mathrm{FrAll}} \\
& K_{z t i}^{\mathrm{Re}} \leq K_{z t i}^{\mathrm{FrAll}} \\
& \sum_{t=1}^{T} Y_{z t i}^{\mathrm{Cl}} \leq 1 \\
& K_{z t i}^{\mathrm{Cl}}+\left(1-Y_{z t i}^{\mathrm{Cl}}\right) M \geq K_{z t i}^{\mathrm{Max}} \\
& K_{z t i}^{\mathrm{Cl}}-M Y_{z t i}^{\mathrm{Cl}} \leq K_{z t i}^{\mathrm{Max}} \\
& K_{z t i}^{\mathrm{Cl}} \leq K_{z t i}^{\mathrm{Max}} \\
& \left(1-Y_{z t i}^{\mathrm{Cl}}\right) M \geq K_{z t i}^{\mathrm{FrAll}} \\
& Z_{z t i}^{\mathrm{New}} n_{i}^{\text {Merge }} \leq \sum_{\hat{i} \in P_{i}^{\text {Merge }}} Y_{z t \hat{i}}^{\mathrm{Cl}} \\
& \left(Y_{z t i j}^{\mathrm{A}}-\sum_{\tau=0}^{t-1} Y_{z \tau i j}^{\mathrm{A}}\right)-1+M\left(1-Y_{z t i}^{\mathrm{NPL}}\right) \geq 0 \\
& \left(Y_{z t i j}^{\mathrm{A}}-\sum_{\tau=1}^{t-1} Y_{z \tau i j}^{\mathrm{A}}\right) \leq Y_{z t i j}^{\mathrm{NPL}} \\
& \left(Y_{z t i j}^{\mathrm{A}}-Y_{z, t-1, i j}^{\mathrm{A}}\right)-1+M\left(1-Y_{z t i j}^{\mathrm{PL}}\right) \geq 0 \\
& Y_{z t i j}^{\mathrm{A}}-Y_{z t i j}^{\mathrm{A}}-Y_{z, t-1, i j}^{\mathrm{A}} \leq Y_{z t i j}^{\mathrm{PL}} \\
& Y_{z t i}^{h} \leq \sum_{i} \sum_{\tau=0}^{t} Y_{z t i j}^{\mathrm{A}} \leq M . Y_{z t j}^{h} \\
& \left(Y_{z t j}^{h}-Y_{z, t-1, j}^{h}-1\right)+M\left(1-Y_{z t j}^{\mathrm{RD}}\right) \geq 0 \\
& Y_{z t j}^{h}-Y_{z, t-1, j}^{h} \leq Y_{z t j}^{\mathrm{RD}} \\
& Y_{z t i}^{\mathrm{Exp}}+Y_{z t i}^{\mathrm{Fr}}+Y_{z t i}^{\mathrm{Cl}} \leq 1 \\
& Y_{z t i}^{\mathrm{Re}}+Y_{z t i}^{\mathrm{Fr}}+Y_{z t i}^{\mathrm{Cl}} \leq 1 \\
& \sum_{j} Y_{z t i j}^{\mathrm{PL}}+Y_{z t i}^{\mathrm{Fr}}+Y_{z t i}^{\mathrm{Cl}} \leq 1 \\
& \forall i, z \\
& \forall i, z \\
& \forall z, t, i \\
& \forall z, t, i \\
& \forall z, t, i \\
& \forall z, t, i \\
& \forall z, t, i \\
& \forall z, t, i \\
& \forall z, t, i \\
& \forall z, t, i \\
& \forall z, t, i \\
& \forall z, t, i \\
& \forall z, t, i \\
& \forall z, t, i \\
& R \in I, \forall z, t, i \\
& \forall z, t, i, j \\
& z, t, i, j \\
& \forall z, t, i, j \\
& z, t, i, j \\
& \forall z, j \\
& \forall z, t, j \\
& \forall z, t, j \\
& \forall z, t, i \\
& \forall z, t, i \\
& \forall z, t, i
\end{aligned}
$$




$$
\begin{aligned}
& \sum_{j} Y_{z t i j}^{\mathrm{NPL}}+Y_{z t i}^{\mathrm{Fr}}+Y_{z t i}^{\mathrm{Cl}} \leq 1 \\
& \forall z, t, i \\
& K_{t i \hat{z}}^{\operatorname{Exp}}=K_{t i \bar{z}}^{\operatorname{Exp}} \quad \forall t, i, \hat{z} \neq \bar{z} \in S_{t} \\
& Z_{t i \hat{z}}^{\mathrm{New}}=Z_{t i \bar{z}}^{\mathrm{New}} \quad \forall t, i, \hat{z} \neq \bar{z} \in S_{t} \\
& Y_{t i \hat{z}}^{\mathrm{Cl}}=Y_{t i \bar{z}}^{\mathrm{Cl}} \\
& \forall t, i, \hat{z} \neq \bar{z} \in S_{\dot{t}} \\
& Y_{t j \hat{z}}^{\mathrm{RD}}=Y_{t j \bar{z}}^{\mathrm{RD}} \\
& \forall t, j, \hat{z} \neq \bar{z} \in S_{\dot{t}} \\
& Y_{t j z}^{\mathrm{Fr}}=Y_{t j \bar{z}}^{\mathrm{Fr}} \\
& \forall t, j, \hat{z} \neq \bar{z} \in S_{\dot{t}} \\
& \forall t, j, \hat{z} \neq \bar{z} \in S_{\dot{t}} \\
& Y_{t j \hat{z}}^{\mathrm{Re}}=Y_{t j \bar{z}}^{\mathrm{Re}} \\
& Y_{t i j \hat{z}}^{\mathrm{NPL}}=Y_{t i j \bar{z}}^{\mathrm{NPL}} \\
& Y_{z t i}^{\mathrm{FrAll}}+\sum_{\tau=0}^{t} Y_{z \tau i}^{\mathrm{Cl}} \leq 1-Y_{z t i}^{\mathrm{ExpW}} \\
& Y_{z t i}^{\text {ExpAll }} \leq \sum_{\tau=0}^{t} K_{z \tau i}^{\text {Exp }} \leq M Y_{z t i}^{\text {ExpAll }} \quad \forall z, t, i \\
& Y_{z t i}^{\mathrm{ExpAll}}-Y_{z t i}^{\mathrm{FrAll}}-\sum_{\tau=0}^{t} Y_{z \tau i}^{\mathrm{Cl}} \leq Y_{z t i}^{\mathrm{ExpW}} \quad \forall z, t, i \\
& Y_{z t i}^{\text {ExpAll }} \geq Y_{z t i}^{\text {ExpW }} \quad \forall z, t, i \\
& \sum_{i} Y_{z t i j}^{v} \leq l_{j} \quad \forall z, t, j \\
& \sum_{j} Y_{z t i j}^{\mathrm{A}} \leq n_{i}^{\mathrm{Max}} \quad \forall z, t, i \\
& \sum_{z}\left(\sum_{i} \mathcal{I}_{z t i}+\sum_{j} \mathcal{D}_{z t j}\right) \leq b_{t} \\
& \mathcal{I}_{z t} \leq \alpha \sum_{r, i, j} C_{z, t-1, r, i j}^{\text {Sale }} X_{z, t-1, r i j} \quad \forall z, t \\
& \sum_{i} X_{z t r i j}+X_{z t r j}^{\mathrm{Unmet}}=d_{z t r j} \quad \forall z, t, r, j \\
& \sum_{r} X_{z t r i j}=X_{z t i j}^{\mathrm{A}} \quad \forall t, i, j . \\
& Y_{z t i}^{\mathrm{Cl}}, Y_{z t i}^{\mathrm{Ou}}, Y_{z t i}^{\mathrm{Fr}}, Y_{z t i}^{\mathrm{Re}}, Y_{z t i}^{\mathrm{FrAll}}, Y_{z t i}^{\mathrm{ExpAll}}, Y_{z t i}^{\mathrm{ExpW}}, Y_{z t i}^{\mathrm{A}} \in\{0,1\} \quad \forall z, t, i \\
& Z_{z t i}^{\mathrm{New}} \in\{0,1\} \quad \forall z, t, i \\
& Y_{z t j}^{\mathrm{Exp}}, Y_{z t j}^{\text {ExpAll }}, Y_{z t j}^{\mathrm{RD}} \in\{0,1\} \quad \forall z, t, j \\
& Y_{z t i j}^{\mathcal{P}}, Y_{z t i j}^{\mathrm{Cl}}, Y_{z t i j}^{\mathrm{NPL}}, Y_{z t i j}^{\mathrm{PL}} \in\{0,1\} \quad \forall z, t, i, j \\
& X_{z t i j}^{\mathrm{A}}, X_{z t i j}, X_{z t i j}^{\mathrm{Unmet}} \geq 0 \quad \forall z, t, i, j \\
& K_{z t i}^{\mathrm{Max}}, K_{z t i}^{\mathrm{Cl}}, K_{z t i}^{\mathrm{Fr}}, K_{z t i}^{\mathrm{Re}}, K_{z t i}^{\mathrm{Exp}}, K_{z t i}^{\mathrm{FrAll}} \geq 0 \quad \forall z, t, i .
\end{aligned}
$$

As summarised at ICOM framework (Figure 1), the following subsections present the cor- 
responding constraints of the model.

\subsubsection{Capacity Volume}

Constraint (3) holds the capacity balance equation according to the initial capacity, new plants, expansion, mothballed, reopened and the shutdown capacity. Constraints (4)-(7) detect each of these decisions via their corresponding binary variables.

\subsubsection{Possibility matrix and production rate}

The fitness of producing product $j$ in plant $i$ is determined via possibility matrix $\gamma_{i j}$ where $\gamma_{i j} \in[0,2]$ and,

- $\gamma_{i j}=1$ indicates that plant $j$ is a normal fit for product $i$

- $0<\gamma_{i j}<1$ product $i$ can be made at plant $j$, but is not the best fit (the production efficiency is between $0 \%$ to $100 \%$ )

- $1<\gamma_{i j} \leq 2$ product $i$ can be made at plant $j$ and with more than normal rates (up to twice faster).

- $\gamma_{i j}=0$ product $i$ cannot be made in plant $j$.

Constraint (8) sets the production capacity based on the fitness of the products.

\subsubsection{Normal and Overutilisation}

A plant $i$ is called overutilised, if it uses more than certain amount of its capacity which is determined by factor $\mu_{i}^{\mathrm{Max}}$. Constraints (9) and (10) force $Y_{z t i}^{\mathrm{Ou}}$ to get 1 if plant $i$ is overutilised in period $t$ under scenario $z$ and 0 , otherwise.

\subsubsection{New Capacity Establishment}

Constraint (11) guarantees that a new plant can be opened at most once within the entire planning horizon.

\subsubsection{Capacity Expansion}

Capacity expansion is limited to a certain number as set in constraint (12) while its lumpiness nature is addressed in constraints (13) and (14). That is, every expansion is limited to a certain range of capacity increase, and not less or more $\left(\vartheta^{\mathrm{E}-\mathrm{min}}\right.$ and $\left.\vartheta^{\mathrm{E}-\mathrm{max}}\right)$. 


\subsubsection{Capacity Mothball}

If mothballing decision is made for plant $i$, i.e., $Y_{z t i}^{\mathrm{Fr}}=1$, then its all available capacity are mothballed: Constraints (15) and (16).

\subsubsection{Capacity Reopen}

Only plants with mothballed capacity can be reopened. The overall mothballed capacity is calculated in (17). Moreover, if reopen decision is made, it opens all the mothballed capacity at once: constraints (18) and (19).

\subsubsection{Capacity Shutdown}

Shutdown of a plant can only happen once, constraint (20), and it can never be reopened if closed down. The corresponding capacity and binary variables are set in constraints (21)-(23). In addition, to avoid sub-optimised solutions, no mothballed capacity can be closed at any time. If a plant is not needed in the future at all, it must be closed down and not mothballed which is set by constraint (24).

\subsection{Relocation and Merge Constraints}

Relocation of a plant means closing a current plant and opening a new one in another location. Similarly, in the case of merging plants, a few plans are closed down to open a new one. Merging portfolios (possible cases) is defined as inputs to the database. It requires investment and lead-time, as well as information on how many ( $\left.n^{\text {Merge }}\right)$ plants and which one of them from the possible potential plants $\left(P^{\text {Merge }}\right)$ could/should be merged to open a new plant as set in (25). For the relocation case (i.e., no merge), $n^{\text {Merge }}=1$.

\subsubsection{Product Development Constraints}

$Y^{\mathrm{NPL}}$ and $Y^{\mathrm{PL}}$ respectively indicate if a product is launched for the first time at all or after a time break. To avoid mixing PL and NPL, the term $Y^{\mathrm{PL}}-Y^{\mathrm{NPL}}$ is applied into the objective function for product relaunch case. Moreover, to formulate whether R\&D has performed or not (i.e., $\left.Y^{\mathrm{RD}}\right)$, the auxiliary binary variable $Y_{z t j}^{h}$ is used to identify if product $j$ has ever been produced in any plants before or not. Constraints (26)-(32) define the above relations and logics. 


\subsubsection{Non-Simultaneous and Non-Anticipative Constraints}

Reopening, expansion and product launch (NPL or PL) can simultaneously be done for the same plant, as well as new plant and new product launch. However, mothballing and reopening, mothballing and expansion, reopening and shutdown, expansion and shutdown, NPL/PL and shutdown and finally NPL/PL and capacity mothball cannot be done simultaneously for the same plant: constraints (33)-(36).

Furthermore, non-anticipative constraints are needed for stochastic models (Sterman 2000), indicating that strategic capacity decisions are irreversible and the decision variables corresponding to the scenarios with the same history have the common value. Let $S_{\dot{t}}$ denote the set of scenarios with a common history and decisions at time $t$. These decisions are capacity expansion, shutdown, new plant establishment, new product launch decision, R\&D, plant mothball, and finally plant reopening as given in (37)-(43).

\subsubsection{Other Constraints}

\section{Workforce Constraints}

Recalling from the objective function, expanded capacities need extra workforce cost if they are operational (not mothballed or closed). In such situations $Y_{z t i}^{\text {ExpAll }}=1$, and 0 otherwise.

- If capacity has been closed down sometime before year $t$ or mothballed and not reopened, then $Y_{z t i}^{\text {ExpAll }}=0$ : constraint (44).

- If the plan has been ever expanded before (i.e., $Y^{\text {ExpAll }}=1$ ), and is not currently mothballed nor closed down, then $Y_{z t i}^{\text {ExpAll }}=1$ : constraints (45) and (46).

- If the plant has never been expanded then $Y_{z t i}^{\text {ExpAll }}=0$ : constraint (47).

\section{Maximum Plant and Maximum Product Constraints}

The company policy might be not to launch each product $j$ in more than a certain maximum number of plants as shown in (48). Likewise, a maximum product constrain as (49) can be applied to limit the maximum number of product types launched in certain plants in each period of time.

\section{Budget Constraints}

Often companies define a maximum annual budget, $b_{t}$, for total investment: (50a). Also, the investment budget can be defined as a proportion of the total annual sales (revenue) as (50b) where $\alpha<1$ is a constant corresponding to proportion of revenue which is assigned for reinvestment. 


\section{Demand and Distribution Constraints}

Unmet demand associated with a penalty is considered in this model and (51) formulates the total production with relate to overall demand. Moreover, all products of year $t$ must be transported within the same period: (52). Note that since this model is a strategic planning one, with a time interval of one year, no inventory term and constraint is required if a first-in-first-out stock management applies (Chen et al. 2002).

\section{Solution and Validation}

This model is a scenario-based stochastic model. After extending the scenarios in the programming (coding in Visual Basic), the model can be reformulated to a mixed integer liner optimisation problem. To validate the model, fourteen hypothetical cases are designed and used, as explained in an ICOM form validation plan in Figure 3. This figure illustrates how all decision outputs of the model and their interactions are validated in this study. Cases 1 to 5 are deterministic cases to validate the model's ability to handle slight, medium and significant capacity changes (increase and decrease). Deterministic cases 6 to 8 are designed to validate model's location decision capability. Case 6 studies the impact of different financial terms (tax, VAT and inflation) on the global decisions, and cases 7 and 8 study the impact of location/relocation capability on capacity volume decisions (relocation versus expansion in demand increase scenarios and relocation versus underutilisation in demand decrease scenarios, respectively). Case 9 and 10 validate stochastic form in capacity volume cases. Although demand is stochastic for the case 9, the expected value of demand is designed to be exactly the same as case 2, in order to study the model's stochastic capability and value of stochastic solutions (VSS) in the increasing demand scenario. Likewise, to study the decreasing demand scenario, case 10 represents a stochastic demand with the same expected value as in the case 5, and calculates VSS in demand decrease scenarios. Similar to Case 7, in case 11 the impacts of global solutions in oppose to local decision (for instance, a new plant in China versus an expansion of a current European plant) is studied in the stochastic form. Cases 12 and 13 validate technology management capability of the model, and respectively volume flexibility and product-mix flexibility. Finally, case 14 validates the model's strategic product development and planning capability. All validation cases are available on request from the corresponding author, but in this paper only cases 5,9 and 11 are reported to validate the capacity volume and location decision capability of the model in deterministic and stochastic forms. Although the model can be used for a wide range of production industries, to be more specific in this paper, 
the general rates and figures for the automotive industry is used for the hypothetical cases, and the input data is adjusted to an average car manufacturer.

Cases 5\&9: In these cases an existing plant in the UK is operational with a slightly increasing demand forecast. To simplify the case, only one product family and one sales region is considered. Table 6 shows the general information about the plant. The VAT is $20 \%$, and no tariff is applied since the plant and sales regions are located in the EU. Unit cost of operations (transportation, dealership and warehouse cost of the product family) in this sales region has been set at $£ 4,000$ per unit.

Running this case in the deterministic form (case 5: deterministic demand similar to the expected value of demand in case 9) and in stochastic form (case 9), the model results in different solutions. The expected demand is more than the normal capacity but just under the maximum capacity of the plant. Table 7 shows two possible solutions (overutilization or expansion) in deterministic case (case 5), overutilization seems to be the best solution. Setting unmet demand penalty as $£ 5 \mathrm{~K}$ per unit and running the model in the deterministic mode and with expected values, the same solution was suggested by the model as well. However, in stochastic mode and including demand scenarios, the model suggests expansion as the best solution. Table 8 compares two possible solutions in stochastic mode and explains why different solutions are suggested for these cases. The result shows VSS in this example which results in a saving of $£ 347 \mathrm{~m}$ over 10 years. 


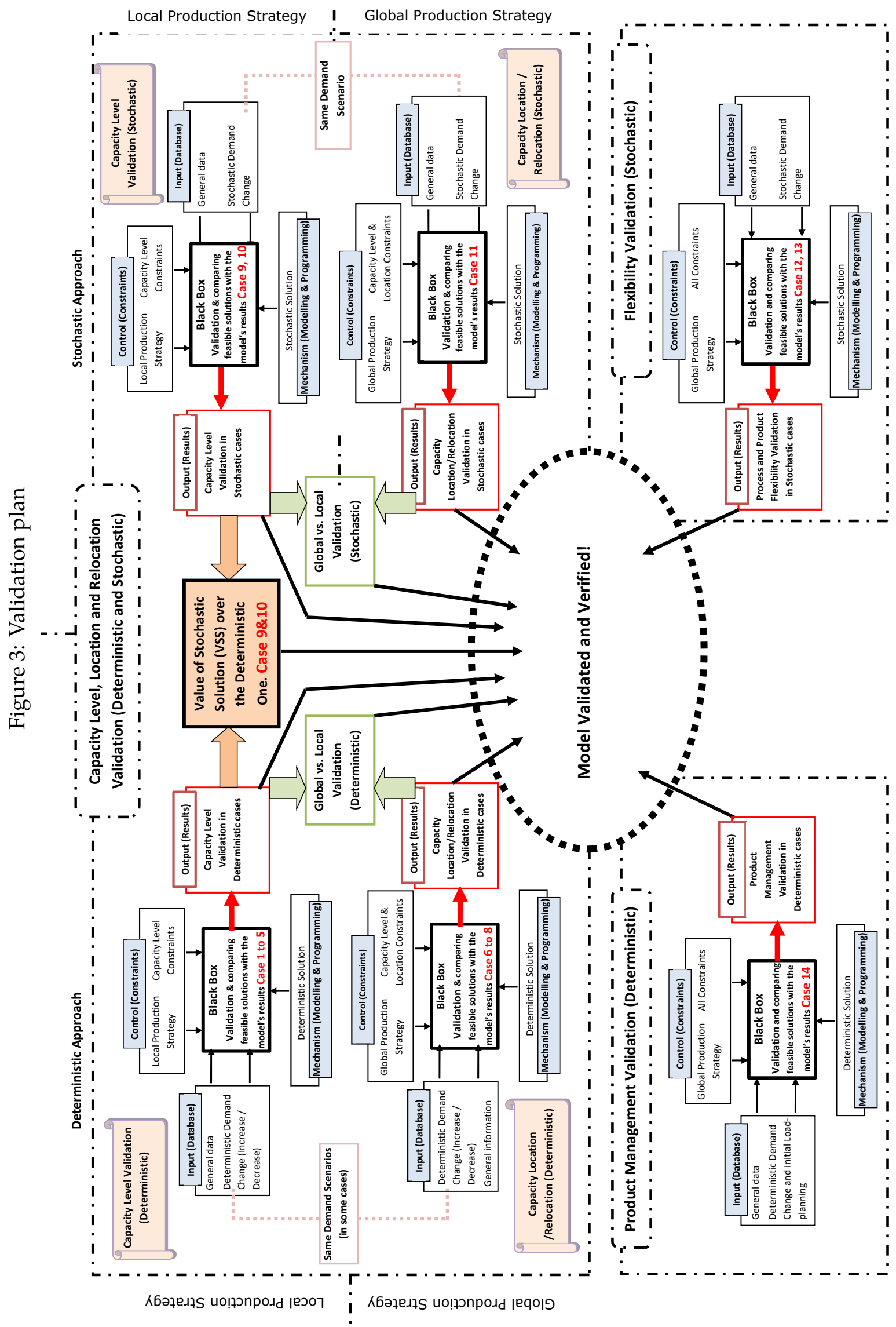


Table 6: General information about the UK plant for the cases 5, 9 and 11

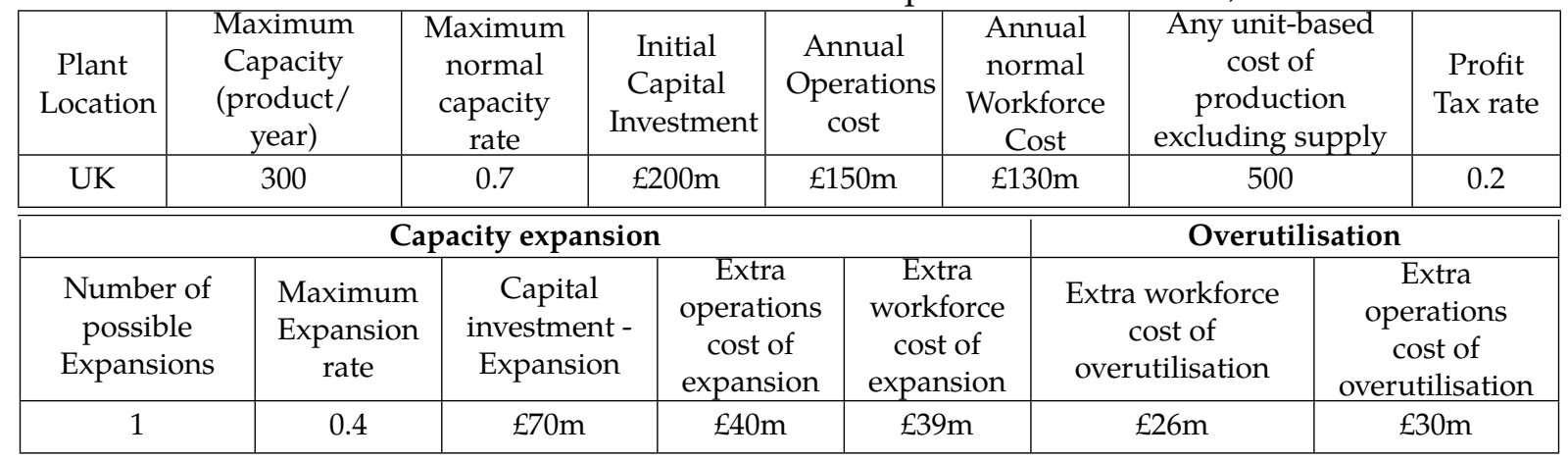

Table 7: Total cost of different solution in deterministic form (case 5)

\begin{tabular}{|c|c|c|c|c|c|c|}
\hline \multirow{2}{*}{ Expansion } & $\begin{array}{c}\text { Expansion } \\
\text { Fixed Cost }\end{array}$ & $\begin{array}{c}\text { Extra } \\
\text { Operations cost } \\
\text { of expansion } \\
\text { (in 7 years) }\end{array}$ & $\begin{array}{c}\text { Extra } \\
\text { Workforce } \\
\text { Cost of } \\
\text { expansion } \\
\text { (in 7 years) }\end{array}$ & $\begin{array}{c}\text { Overutilisation } \\
\text { fixed cost } \\
\text { (in 7 years) }\end{array}$ & $\begin{array}{c}\text { Overutilisation } \\
\text { Work force } \\
\text { cost } \\
\text { (in 7 years) }\end{array}$ & Sum \\
\cline { 2 - 7 } & 0.4 & $£ 70 \mathrm{~m}$ & $£ 40 \mathrm{~m}$ & $£ 39 \mathrm{~m}$ & $£ 26 \mathrm{~m}$ & $£ 30 \mathrm{~m}$ \\
\hline \hline \multirow{2}{*}{ Overutilisation } & Overutilisation fixed cost in 10 years & \multicolumn{2}{|c|}{ Overutilisation Workforce cost in 10 years } & Sum \\
\cline { 2 - 7 } & \multicolumn{2}{|r|}{$£ 260 \mathrm{~m}$} & \multicolumn{2}{|c|}{$£ 300 \mathrm{~m}$} & $£ 560 \mathrm{~m}$ \\
\hline
\end{tabular}

Table 8: Total cost of different solutions in stochastic form (case 9) and VSS

\begin{tabular}{|c|c|c|c|c|c|c|c|c|c|c|c|c|c|}
\hline \multicolumn{6}{|c|}{ Expansion Solution } & \multirow[b]{2}{*}{\begin{tabular}{l}
\multicolumn{1}{c}{ Scenario } \\
$p=$ probability \\
GP=Unit Gross \\
Profit (sales price- \\
unit operations \\
and supply cost)
\end{tabular}} & \multicolumn{7}{|c|}{$\begin{array}{l}\text { Overutilisation and No Expansion } \\
\text { Solution Capacity is capped to } 3\end{array}$} \\
\hline 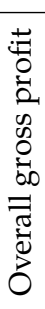 & 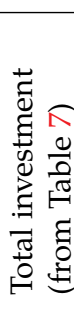 & 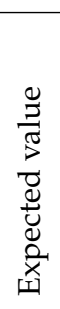 & 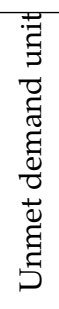 & 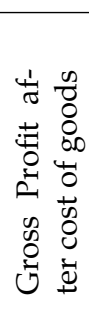 & 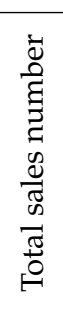 & & 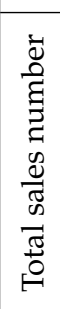 & 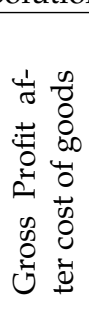 & 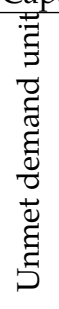 & 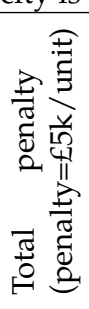 & 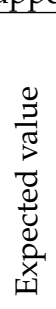 & 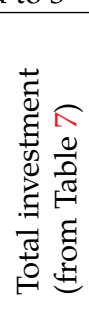 & 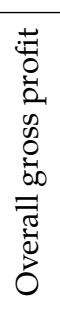 \\
\hline \multirow{3}{*}{$\begin{array}{l}\text { ह } \\
\text { สิ } \\
\text { प्र }\end{array}$} & \multirow{3}{*}{ 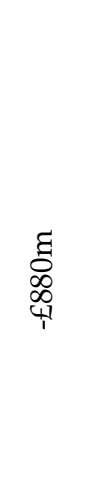 } & 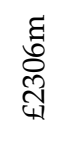 & 0 & 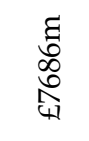 & $\begin{array}{l}\text { तू } \\
\stackrel{\mathrm{N}}{\mathrm{N}}\end{array}$ & $\begin{array}{l}p=30 \% \\
G P=£ 3 k \\
£(29-26) \mathrm{k}\end{array}$ & 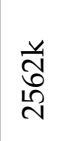 & 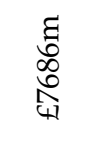 & 0 & 0 & 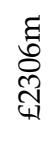 & \multirow{3}{*}{ 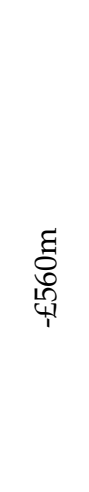 } & \multirow{3}{*}{ 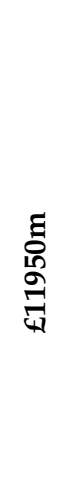 } \\
\hline & & 胥 & 0 & $\begin{array}{l}\text { ह્ } \\
\text { 竎 } \\
\text { 常 }\end{array}$ & $\begin{array}{l}\breve{2} \\
\infty \\
\infty \\
\infty \\
\infty\end{array}$ & $\begin{array}{l}p=50 \% \\
G P=£ 5 k \\
£(31-26) \mathrm{k}\end{array}$ & 峁 & 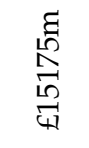 & 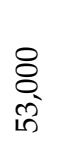 & 萬 & 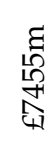 & & \\
\hline & & 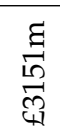 & 0 & 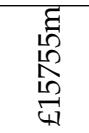 & $\frac{\ddot{m}}{\stackrel{\vec{n}}{m}}$ & $\begin{array}{l}p=20 \% \\
G P=£ 5 k \\
£(31-26) \mathrm{k}\end{array}$ & 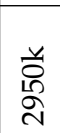 & 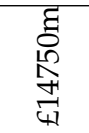 & $\begin{array}{l}8 \\
\stackrel{\text { ¿े }}{ }\end{array}$ & 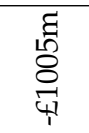 & 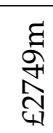 & & \\
\hline
\end{tabular}


Case11: This case is designed to compare local and global cases and calculates the value of global solutions (VGS). The expected demand volume remains the same as the one in cases 5 and 9, but different sales regions (UK, US and China) are introduced with 3 different demand scenarios for each region given in Table 9. In this case, besides the existing plant in the UK (Table 6), an optional new plant in China can also be opened by the model as an alternative solution to overutilisation and expansion of the UK plant. Investments and financial figures for the plant in China are listed in Table 10. Sales price, transportation costs and tariff rates are shown in Table 11. Running the model in global mode for this simple case, it suggests opening a new plant in China and utilise it at normal level, rather than expanding the plant in the UK. Although as Table 12 shows this option needs $£ 1,780 \mathrm{~m}$ investment in fixed and variable costs over 9 years (as oppose to $£ 924 \mathrm{~m}$ for expanding the existing plant in the UK), major savings on tariff and transportation fees justifies this new capacity establishment. Table 12 illustrates that the value of global solution (VGS) in this case is $£ 4,189$ m over 9 years of planning. This result shows the importance of global location decisions embedded in a capacity management model.

Table 9: Demand scenarios in different sales region (expected demand is similar to case 5 \& 9)

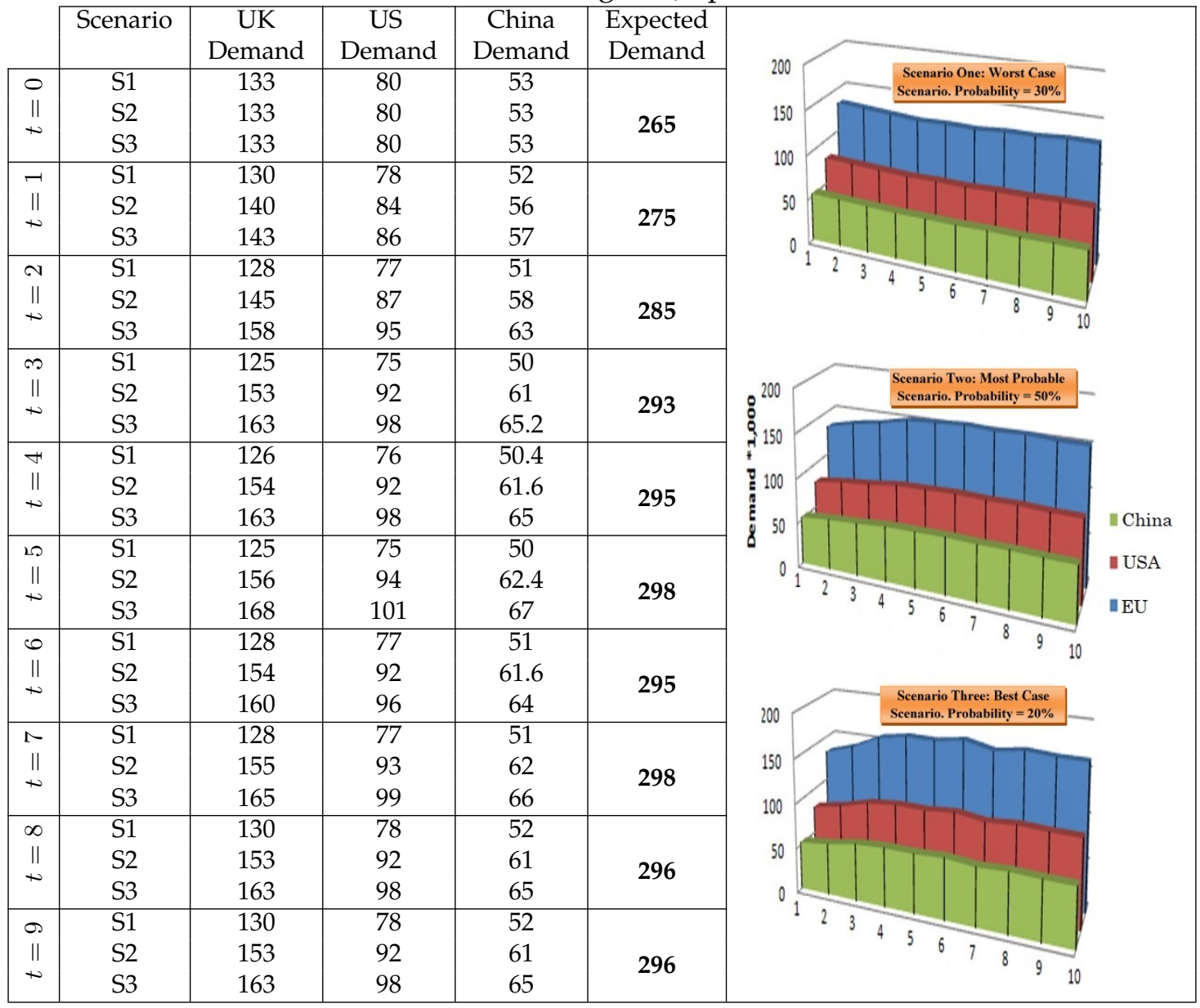


Table 10: Input data for the optional plant in China

\begin{tabular}{|c|c|c|c|c|c|c|c|}
\hline $\begin{array}{c}\text { Plant } \\
\text { Location }\end{array}$ & $\begin{array}{c}\text { Maximum } \\
\text { capacity } \\
\text { (product/ } \\
\text { year) }\end{array}$ & $\begin{array}{l}\text { Maximum } \\
\text { normal } \\
\text { capacity } \\
\text { rate }\end{array}$ & $\begin{array}{c}\text { Initial } \\
\text { capital } \\
\text { Investment }\end{array}$ & $\begin{array}{l}\text { Annual } \\
\text { operations } \\
\text { cost }\end{array}$ & $\begin{array}{c}\text { Annual } \\
\text { normal } \\
\text { workforce } \\
\text { Cost }\end{array}$ & $\begin{array}{l}\text { Any unit-based } \\
\text { cost of } \\
\text { production } \\
\text { excluding } \\
\text { supply }\end{array}$ & $\begin{array}{l}\text { Profit } \\
\text { tax rate }\end{array}$ \\
\hline China & 200,000 & 0.8 & $£ 200 \mathrm{~m}$ & $£ 100 \mathrm{~m}$ & $£ 60 \mathrm{~m}$ & $£ 500 /$ unit & 0 \\
\hline \multicolumn{5}{|c|}{ Capacity Expansion } & \multicolumn{3}{|c|}{ Overutilisation } \\
\hline $\begin{array}{l}\text { Number } \\
\text { of pos- } \\
\text { sible } \\
\text { Expan- } \\
\text { sions }\end{array}$ & $\begin{array}{l}\text { Maximum } \\
\text { expansion } \\
\text { rate }\end{array}$ & $\begin{array}{l}\text { Capital in- } \\
\text { vestment } \\
\text { for } \\
\text { expansion }\end{array}$ & $\begin{array}{c}\text { Extra } \\
\text { annual } \\
\text { opera- } \\
\text { tions cost } \\
\text { in case of } \\
\text { expan- } \\
\text { sion }\end{array}$ & $\begin{array}{c}\text { Extra } \\
\text { annual } \\
\text { work- } \\
\text { force cost } \\
\text { in case of } \\
\text { expan- } \\
\text { sion }\end{array}$ & $\begin{array}{l}\text { Capital in- } \\
\text { vestment } \\
\text { for } \\
\text { overutili- } \\
\text { sation }\end{array}$ & $\begin{array}{c}\text { Extra } \\
\text { operations cost } \\
\text { in case of } \\
\text { overutilisation }\end{array}$ & $\begin{array}{c}\text { Extra } \\
\text { workforce } \\
\text { cost in case } \\
\text { of overutili- } \\
\text { sation }\end{array}$ \\
\hline 1 & 0.4 & $£ 50 \mathrm{~m}$ & $£ 20 \mathrm{~m}$ & $£ 15 \mathrm{~m}$ & $£ 5 \mathrm{~m}$ & $£ 5 \mathrm{~m}$ & $£ 10 \mathrm{~m}$ \\
\hline
\end{tabular}

Table 11: Sales price, transportation costs and tariff rates for different plants in different sales regions

Sales Price in different sales regions

Cost of transportation from the UK plant to dealers within different sales regions

Cost of transportation from China plant to dealers within different sales regions

Tariff rates for products coming from the UK plant to different sales regions

Tariff rates for products coming from China plant to different sales regions

\begin{tabular}{|c|c|c|}
\hline EU & USA & China \\
$£ 31,000$ & $£ 32,000$ & $£ 33,000$ \\
$£ 1,000$ & $£ 4,000$ & $£ 8,000$ \\
$£ 4,000$ & $£ 6,000$ & $£ 2,000$ \\
$0 \%$ & $10 \%$ & $20 \%$ \\
$20 \%$ & $20 \%$ & $0 \%$ \\
\hline
\end{tabular}

Table 12: Total difference between local capacity options (case 9) and global mode (case 11)

\begin{tabular}{|c|c|c|c|c|c|c|c|c|c|c|c|}
\hline \multirow{3}{*}{ 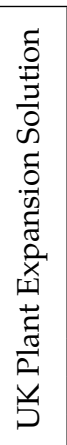 } & 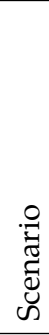 & 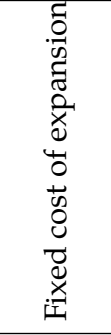 & \multicolumn{2}{|c|}{ 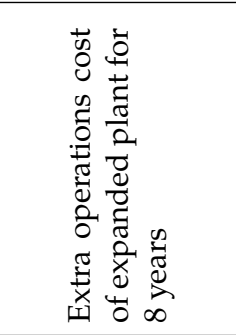 } & \multicolumn{2}{|c|}{ 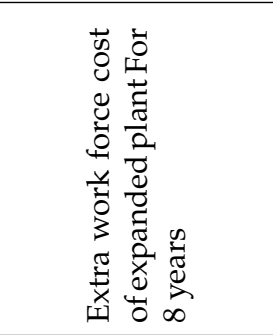 } & 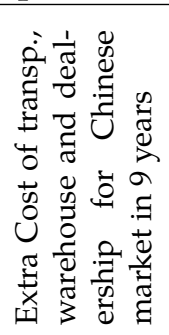 & 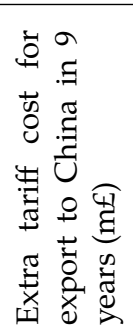 & 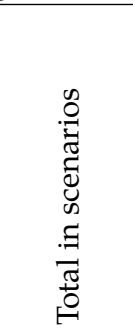 & 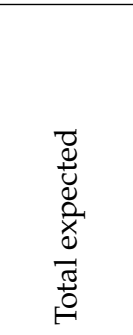 & \multirow[t]{4}{*}{ g్ } \\
\hline & $\begin{array}{l}\mathrm{S} 1 \\
\mathrm{~S} 2\end{array}$ & \multirow[t]{2}{*}{$£ 80 \mathrm{~m}$} & \multirow{2}{*}{\multicolumn{2}{|c|}{$£ 480 \mathrm{~m}$}} & \multirow{2}{*}{\multicolumn{2}{|c|}{$£ 364 \mathrm{~m}$}} & $\begin{array}{l}£ 2,756 \mathrm{~m} \\
£ 3,268 \mathrm{~m}\end{array}$ & $\begin{array}{l}£ 3,032 \mathrm{~m} \\
£ 3,594 \mathrm{~m}\end{array}$ & $\begin{array}{l}£ 6,712 \mathrm{~m} \\
£ 7,786 \mathrm{~m}\end{array}$ & $£ 7,546 \mathrm{~m}$ & \\
\hline & S3 & & & & & & $£ 3,463 \mathrm{~m}$ & $£ 3,810 \mathrm{~m}$ & $£ 8,197 \mathrm{~m}$ & & \\
\hline \multirow[t]{3}{*}{ 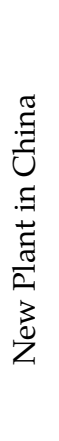 } & 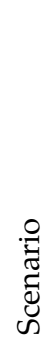 & 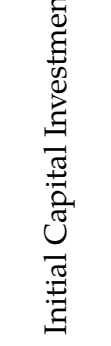 & 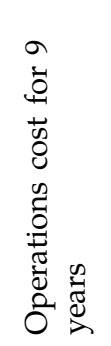 & 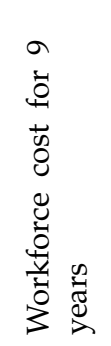 & 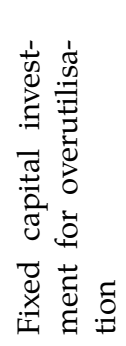 & 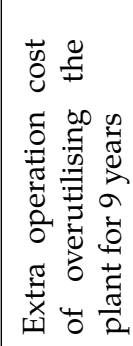 & 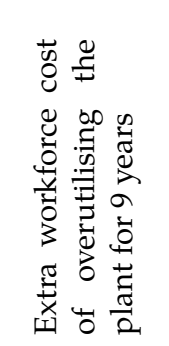 & 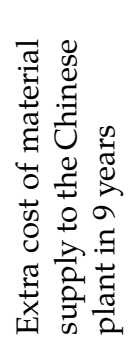 & 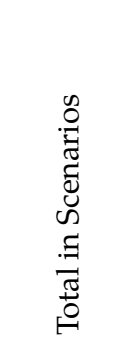 & 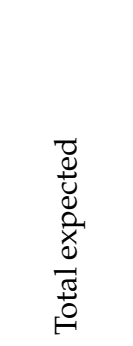 & \\
\hline & $\begin{array}{l}\mathrm{S} 1 \\
\mathrm{~S} 2 \\
\end{array}$ & \multirow[t]{2}{*}{$£ 200 \mathrm{~m}$} & \multirow[t]{2}{*}{$£ 900 \mathrm{~m}$} & \multirow[t]{2}{*}{$£ 540 \mathrm{~m}$} & \multirow[t]{2}{*}{$£ 5 \mathrm{~m}$} & \multirow[t]{2}{*}{$£ 45 \mathrm{~m}$} & \multirow[t]{2}{*}{$£ 90 \mathrm{~m}$} & $\begin{array}{l}11,378 \mathrm{~m} \\
£ 1,634 \mathrm{~m}\end{array}$ & $\begin{array}{l}£ 3,248 \mathrm{~m} \\
£ 3,504 \mathrm{~m}\end{array}$ & \multirow[t]{2}{*}{$£ 3,357 \mathrm{~m}$} & $\stackrel{\vec{\mu}}{\frac{\pi}{\nu}}$ \\
\hline & - & & & & & & & $£ 1,732 \mathrm{~m}$ & $£ 3,602 \mathrm{~m}$ & & \\
\hline
\end{tabular}




\section{Verification of the model: The case of Toyota UK ${ }^{1}$}

Having two assembly lines in Burnaston, Toyota UK (TMUK), with a maximum capacity of 285,000 vehicles per year, was one of the top 5 car manufacturers in Britain in 2009 (Bekker 2010). During the last economy recession in 2010, TMUK forced to mothball one of their production lines towards the end of 2010 (Lea 2010). Using publicly available data and simple assumptions, this section employs our model in this real scale case. Financial information of TMUK (FAME Database. 2010) reveals that after the recession in 2008, TMUK lost almost $£ 1$ billion in annual sales, from $£ 2.774$ billion in 2007 to $£ 1.82$ billion in 2009 . In the first months of 2010, Toyota faced with another disaster, "safety problems", which forced the company to recall around 8 million passenger cars all over the world, including around 200,000 cars in the UK (Telegraph 2010). To react, TMUK firstly scaled down its second production line in Burnaston to one shift in September 2010, and then mothballed this line by the end of 2010 (Lea 2010). Although no labour layoff happened at the time, TMUK supported their mothballed policy, stating that having one fully utilised production line is more feasible than having two underutilised lines (Bawden and Lewis 2010). Table 13 shows total sales and operations cost of the company.

Table 13: Total annual cost of the company (in Emillion)

\begin{tabular}{rrrrrrrrr}
\hline & $\mathbf{2 0 0 2}$ & $\mathbf{2 0 0 3}$ & $\mathbf{2 0 0 4}$ & $\mathbf{2 0 0 5}$ & $\mathbf{2 0 0 6}$ & $\mathbf{2 0 0 7}$ & $\mathbf{2 0 0 8}$ & $\mathbf{2 0 0 9}$ \\
\hline TMUK car production $(\times 1,000)$ & 212 & 211 & 245 & 263 & 282 & 275 & 164 & 127 \\
Supply costs (Total) & 1,004 & 1,594 & 1,609 & 1,823 & 1,800 & 1,942 & 1,419 & 1,267 \\
Total remuneration & 129.2 & 165.5 & 175.9 & 179.2 & 172.1 & 162.4 & 154.6 & 124.1 \\
Depreciation & 135.6 & 97.4 & 114.1 & 103 & 115.1 & 115.5 & 106.1 & 87.2 \\
Operation expenses & 33.3 & 39.9 & 37.8 & 39.2 & 39.2 & 33.4 & 31.4 & 22 \\
Other costs of operations & 198.8 & 307.6 & 267.8 & 358.4 & 399.7 & 484.8 & 476.9 & 363 \\
\hline Source: (FAME Database. 2010) and (Toyota Motor Annual Report. 2010, Toyota Motor Corporation. 2010)
\end{tabular}

To generate scenarios only the data and information available prior to mothball decision (end of 2010) was used in this case. The sales estimation for Europe expected a 19.2\% decline, to 858,000 units, and Toyota's total production in the EU was expected to decline by $10.2 \%$, to 433,000 units in 2010 (Ruddick 2010). Despite such downtime, what was promising for TMUK sales was the fact that the company was preparing to launch Toyota Auris Hybrid model in the Burnaston facility in 2010, which had been expected to be a game-changer for TMUK. Toyota's first forecast for 2011 fiscal year (ending March 31, 2011) were a vehicle sales of 7.29 million units (Toyota Motor Corporation. 2010). However, due to some evidence of the recession recovery

\footnotetext{
${ }^{1}$ A verification with the case of Jaguar Land Rover (JLR) investment in China is also available on request from the authors.
} 
signs by early 2010, Toyota revised its sales forecast to 7.41m units for 2011 (Costea 2010). Based on these facts and information, three main scenarios for 2010 and 2011 were defined in this study, comprising demand decrease, stable demand and demand increase. In an interview with TMUK senior management team at the time (2009), demand decrease was found to be the most probable scenario (with $50 \%$ probability allocation), in which a $5 \%$ and a $10 \%$ sales reductions were estimated for 2010 and 2011, respectively. The other 50\% probability was divided equally to stable demand scenario $(p=25 \%)$ and a demand increase scenario with $5 \%$ sales increase estimations for 2010 and 2011.

This case consists of 3 products, 2 production lines, 3 scenarios, 3 time periods, and 2 stages. To set the input parameters data from table 13 was used and a two-stage scholastic version of our model was employed for the case. This example contains three binary decision variables of capacity under-utilisation, capacity mothball, and capacity shutdown, as well as two continuous decision variable of production lot allocations to both lines. Unmet demand penalty for each of three cars was set to their gross profit margin plus a fixed penalty as called "trust image penalty". Other decision variables, input parameters and constraints that were not tied to capacity volume module were turned off and The model suggests to mothball the second production line and fully utilise the first production lien in 2010 which verifies the model with the actual decision taken by TMUK at the time. In the second run, the model was given the data available in 2007 and 2008, which shows early signs of global recession, and the model suggested a mothball in 2009 , which could have saved the company over $£ 10$ m of operations cost of running both lines under-utilised for one extra year, before the actual mothball in 2010.

\section{Discussion and Conclusion}

Although many peers had raised a need to apply external and market uncertainty into capacity management models, as Table 14 shows, the use of stochastic programming in embedding uncertainty into capacity design and planning has only taken off very recently [after 2009] while most of the models in the field were deterministic before that. However, comparing Tables 14 and 15 indicates that the stochastic models are still not as multi-functional and versatile as the deterministic ones, and they can handle less capacity decision terms than what their deterministic counterparts can manage. Thus, it shows although existing stochastic capacity management models may be able to handle market scenarios, they lack taking a global and comprehensive approach into their decision terms, which limits them to limited applications and can cause sub-optimal solutions. It shows a gap for a more holistic and integrated capacity management 
model in stochastic form that can handle market uncertainty, while not compromising on decision terms and boundary conditions. This work aimed to address this gap and to develop a stochastic model that can simultaneously handle capacity level management (in increase and decrease scenarios and in realistic terms of sight, moderate and significant changes), capacity location, relocation merge and decomposition, product development ( $\& D$, new product introduction and re-launch cases), and technology management (product and process flexibility and technology life-cycles). Cumbersome data requirements of stochastic models and the size of the extended models (which causes long solution times) have been often blamed for limited decision terms in the stochastic practices (Snyder 2006; Tenhiälä 2011). However, this paper showed these limitations can be overcome by the use of enumerated scenarios, detailed constrains, and right programming approach, which all can reduce the size of the final model to a manageable scale. Enumerated scenario approach causes no limitation to the strategic capacity management modelling, as after all, the number of possible scenarios is inherently limited in strategic planning. A systematic model development approach, which is often called open-box validation was adopted, using ICOM logic (Matta et al. 2005), as explained in Section 1 and employed in Section 3. Using the same logic, a holistic validation plan was developed in ICOM format and employed to test and validate the model. As frequently raised by other authors in this area, verification of the resource management models with real-scale and industrial cases has yet been remained widely unattempted (Hammami et al. 2008; Julka et al. 2007), and there is still a widespread need in the field to make managers and industrial decision-makers aware of what operation research (OR) and optimisation-based capacity management models have to offer to the world of practice (Ackermann et al. 2014). This paper briefly applied this model to the case of Toyota-UK (and the case of Jaguar-Land Rover is also available upon request from the corresponding author), in an attempt not only to verify the model in real scales, but also to illustrate the potential contribution such models can offer to strategic decision-making in practice.

\section{Appendix}




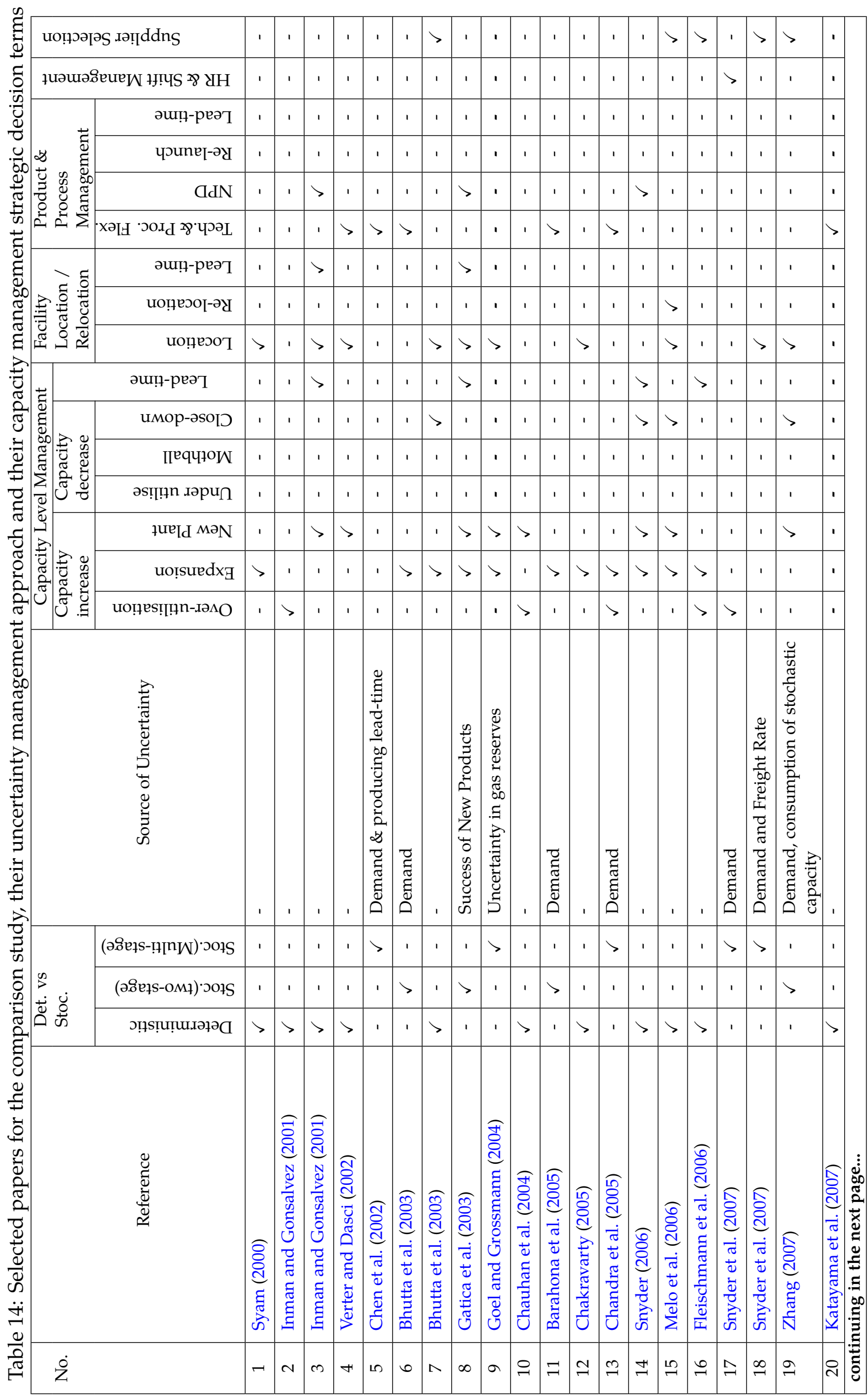




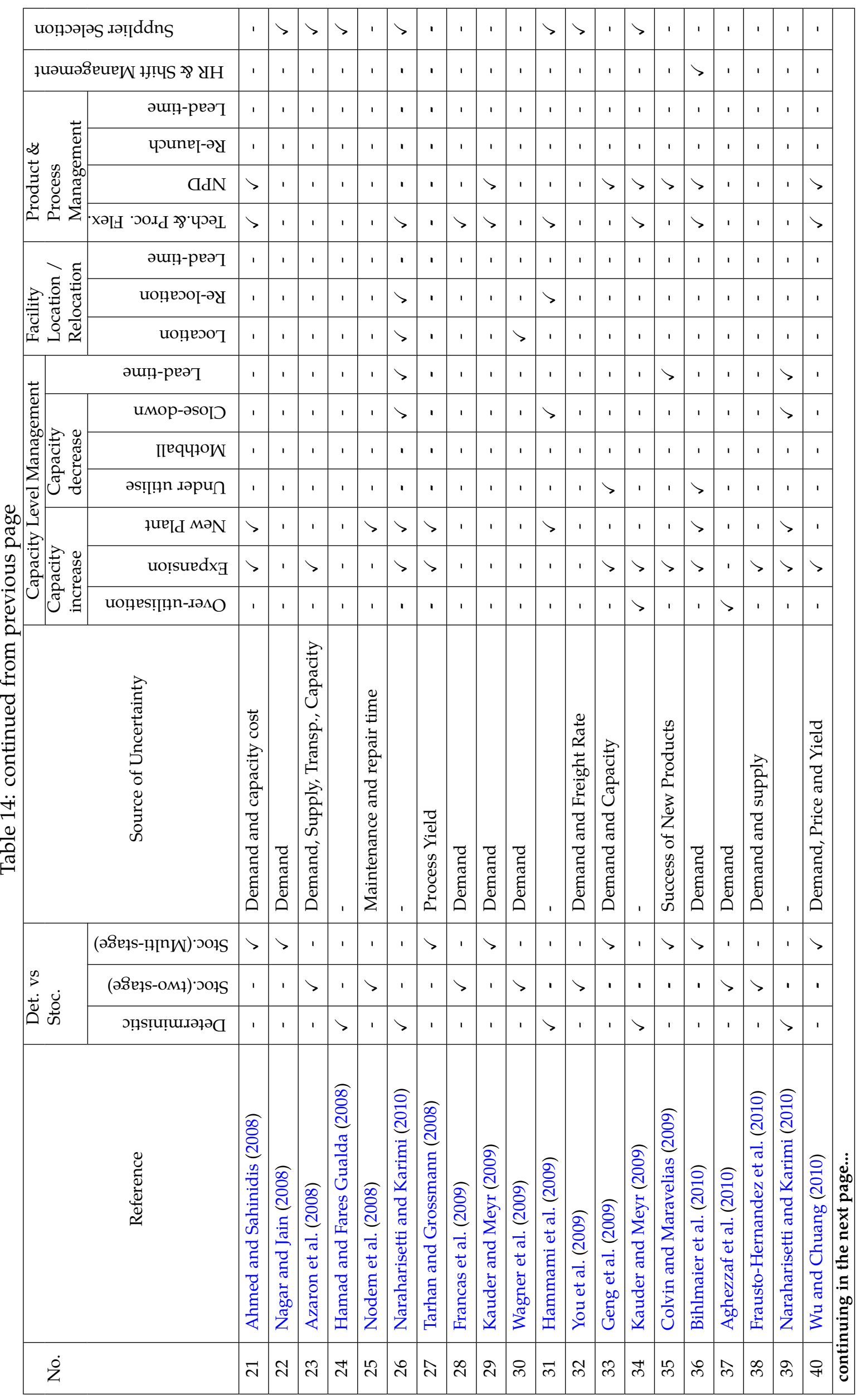




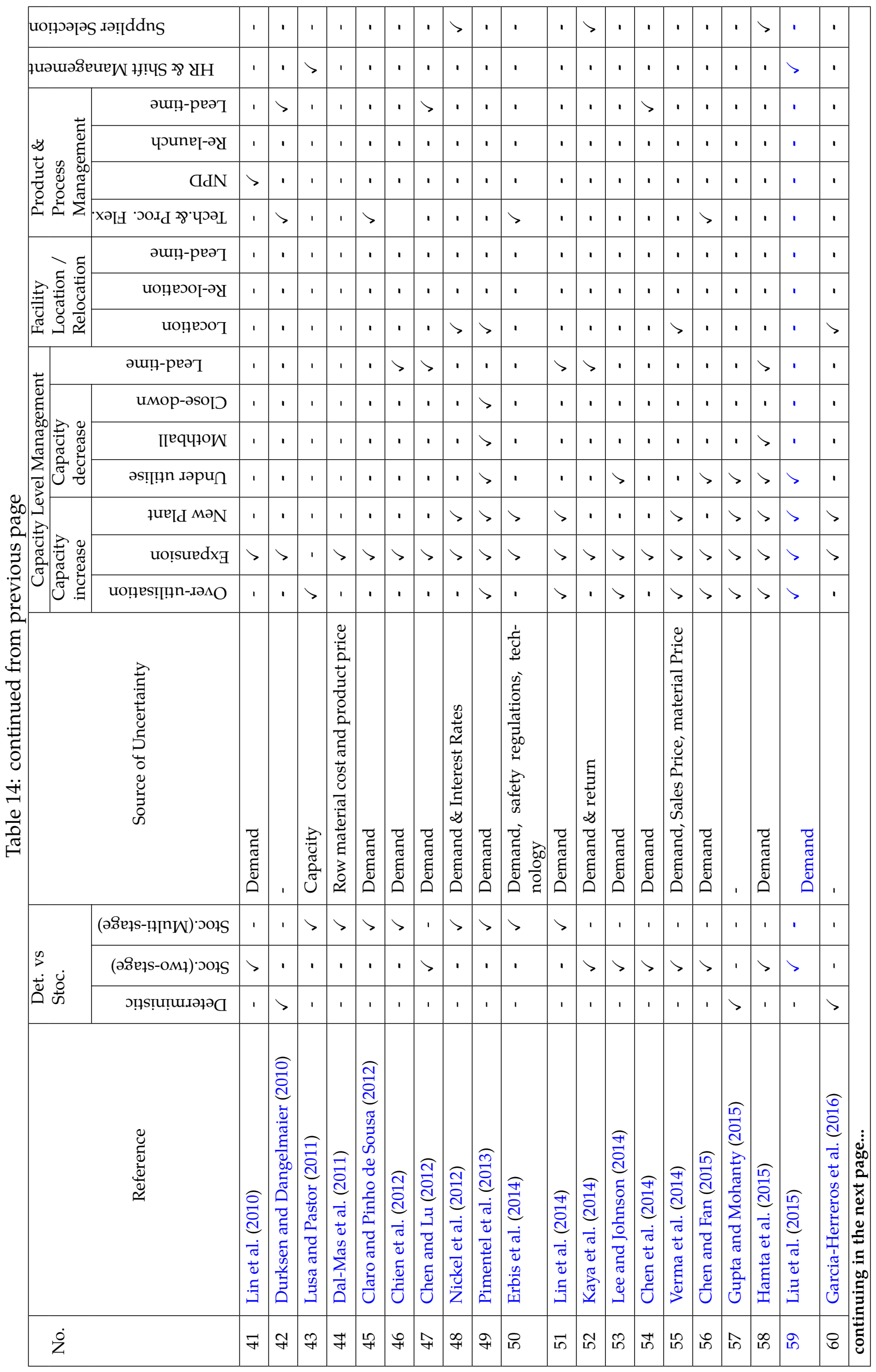




\begin{tabular}{|c|c|c|c|c|c|c|c|c|c|c|}
\hline \multicolumn{2}{|c|}{ 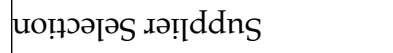 } & $>$ & . & $>$ & . & . & . & ' & $>$ & \\
\hline \multicolumn{2}{|c|}{ 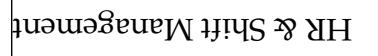 } & ' & ' & ' & ' & ' & ' & ' & ' & ' \\
\hline \multirow{4}{*}{ 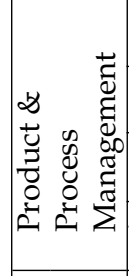 } & әщ!̨-реәт & $>$ & ' & ' & ' & ' & $>$ & ' & ' & \\
\hline & чગune[-әу & ' & ' & ' & ' & ' & , & ' & ' & \\
\hline & GdN & $>$ & ' & ' & ' & ' & ' & ' & ' & \\
\hline & 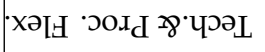 & ' & $>$ & ' & $>$ & $>$ & $>$ & $>$ & ' & ' \\
\hline \multirow{3}{*}{ 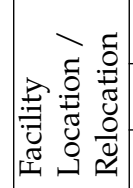 } & әш!?-реәт & ' & ' & ' & ' & ' & ' & ' & ' & \\
\hline & иоџ̣еәо-әу & ' & ' & ' & ' & ' & ' & ' & , & \\
\hline & uоп̣еวот & ' & ' & ' & ' & ' & ' & $>$ & $>$ & \\
\hline \multirow{4}{*}{ 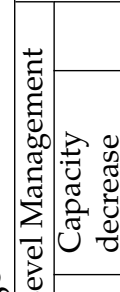 } & әш!̣-реәт & ' & ' & . & ' & $>$ & $>$ & ' & ' & ' \\
\hline & имор-әsоIว & ' & ' & 1 & $>$ & $>$ & $>$ & ' & ' & ' \\
\hline & IIеqчłо & ' & ' & ' & ' & ' & , & ' & ' & \\
\hline & 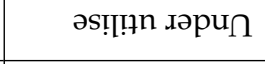 & ' & $>$ & ' & $>$ & $>$ & $>$ & $>$ & ' & \\
\hline & ҰUए Id MəN & ' & $>$ & ' & ' & ' & $>$ & $>$ & $>$ & ' \\
\hline 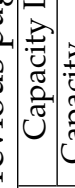 & uoṭsuedxH & ' & $>$ & $>$ & $>$ & $>$ & $>$ & $>$ & ' & $>$ \\
\hline$\tilde{u}^{\pi} . \Xi$ & uo!̣es!̣!̣n-лəАО & ' & $>$ & $>$ & $>$ & $>$ & $>$ & $>$ & $>$ & ' \\
\hline & 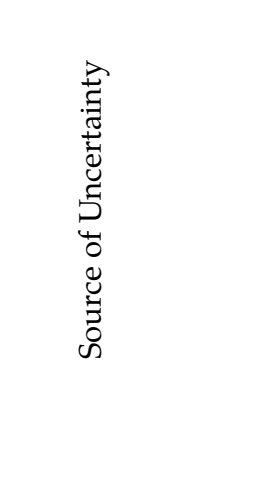 & 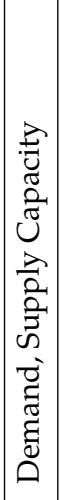 & 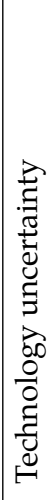 & 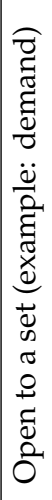 & 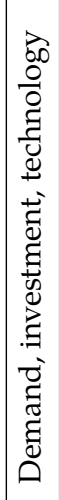 & 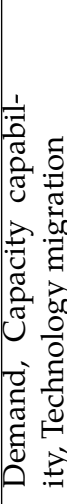 & 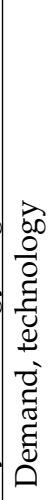 & 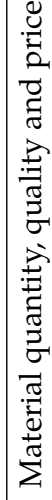 & . & 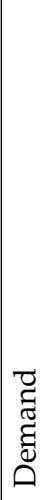 \\
\hline \multirow{3}{*}{ 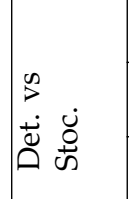 } & 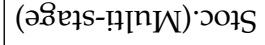 & $>$ & $>$ & ' & $>$ & ' & ' & ' & ' & $>$ \\
\hline & 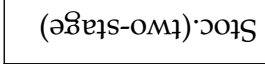 & , & ' & $>$ & ' & ' & $>$ & $>$ & $>$ & ' \\
\hline & 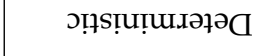 & 1 & ' & ' & ' & $>$ & ' & ' & ' & ' \\
\hline \multicolumn{2}{|r|}{ 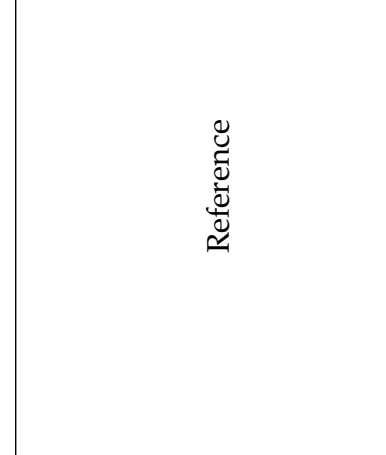 } & 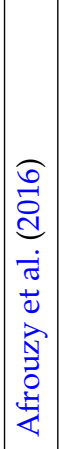 & 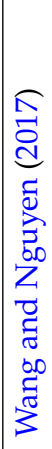 & 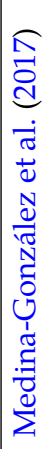 & 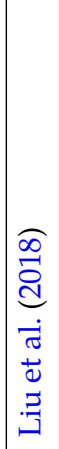 & 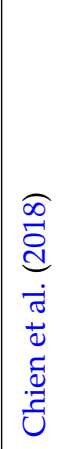 & 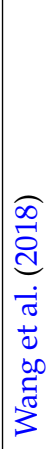 & 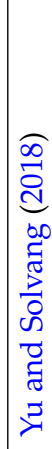 & 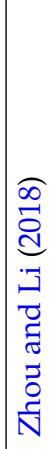 & 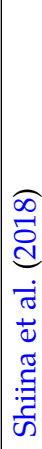 \\
\hline$\dot{z}$ & & $\overrightarrow{6}$ & ชิ & 8 & オु & 뭉 & 8 & $\hat{\sigma}$ & $\infty$ & g \\
\hline
\end{tabular}


Table 15: More detailed terms and constraints in the selected Capacity management models

\begin{tabular}{|c|c|c|c|c|c|c|c|c|c|c|c|c|c|c|c|c|c|c|c|}
\hline \multirow[b]{2}{*}{ 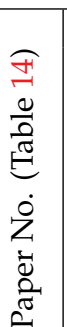 } & \multicolumn{8}{|c|}{ Cost Parameters } & \multicolumn{6}{|c|}{ Financial Parameters } & \multicolumn{2}{|c|}{ Prod./Proc. } & \multirow[b]{2}{*}{ 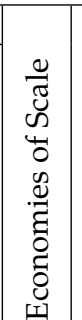 } & \multirow[b]{2}{*}{ 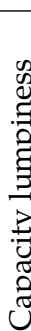 } & \\
\hline & 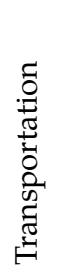 & 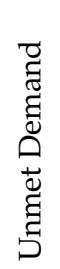 & $\begin{array}{l}\overrightarrow{0} \\
0 \\
0 \\
0 \\
0 \\
0 \\
\tilde{D} \\
0 \\
0 \\
0\end{array}$ & 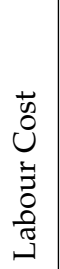 & 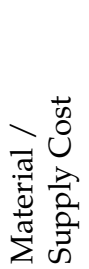 & 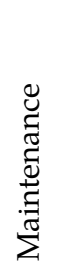 & 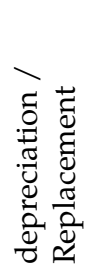 & 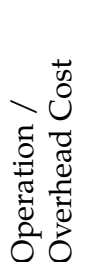 & 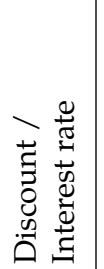 & 蔍 & $\frac{\overrightarrow{2}}{\overrightarrow{2}}$ & $\$$ & 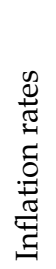 & $\begin{array}{l}\widetilde{L} \\
\text { s. } \\
\end{array}$ & 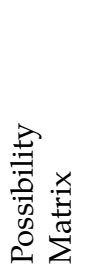 & 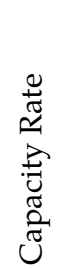 & & & \\
\hline
\end{tabular}

\begin{tabular}{|c||c|c|c|c|c|c|c|c|c|c|c|c|c|}
\hline 1 & $\checkmark$ & - & $\checkmark$ & $\checkmark$ & - & - & - & - & - & - & - & - & - \\
\hline 2 & - & $\checkmark$ & - & - & - & - & - & - & - & - & - & - & - \\
\hline 3 & - & - & $\checkmark$ & $\checkmark$ & $\checkmark$ & - & $\checkmark$ & $\checkmark$ & $\checkmark$ & $\checkmark$ & - & - & - \\
\hline 4 & $\checkmark$ & - & $\checkmark$ & - & - & - & - & $\checkmark$ & - & - & - & - & - \\
\hline 5 & - & - & - & - & - & - & - & - & - & - & - & - & - \\
\hline 6 & - & $\checkmark$ & - & - & - & - & - & - & - & - & - & - & - \\
\hline 7 & $\checkmark$ & - & $\checkmark$ & - & - & $\checkmark$ & $\checkmark$ & $\checkmark$ & - & - & $\checkmark$ & - & $\checkmark$ \\
\hline 8 & - & $\checkmark$ & $\checkmark$ & - & $\checkmark$ & $\checkmark$ & - & $\checkmark$ & $\checkmark$ & - & - & - & - \\
\hline 9 & $\checkmark$ & - & - & - & - & - & - & - & $\checkmark$ & - & - & - & -
\end{tabular}

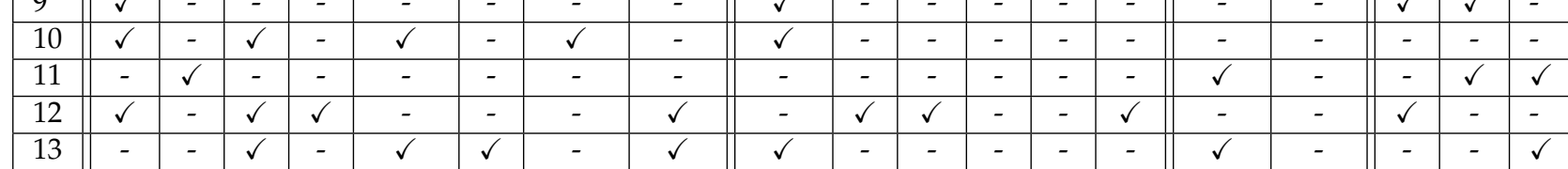

\begin{tabular}{|c|c|c|c|c|c|c|c|c|c|c|c|c|c|c|c|c|c|c|c|}
\hline 13 & - & - & $\checkmark$ & - & $\checkmark$ & $\checkmark$ & - & $\checkmark$ & $\checkmark$ & - & - & - & - & - & $\checkmark$ & - & - & - & $\checkmark$ \\
\hline 14 & $\checkmark$ & $\checkmark$ & $\checkmark$ & - & - & - & - & $\checkmark$ & - & - & - & - & - & - & $\checkmark$ & - & $\checkmark$ & $\checkmark$ & - \\
\hline 15 & $\checkmark$ & - & $\checkmark$ & - & - & - & - & $\checkmark$ & $\checkmark$ & - & - & - & - & - & $\checkmark$ & $\checkmark$ & $\checkmark$ & $\checkmark$ & $\checkmark$ \\
\hline 16 & $\checkmark$ & - & $\checkmark$ & - & $\checkmark$ & - & - & $\checkmark$ & $\checkmark$ & - & - & - & - & - & $\checkmark$ & - & $\checkmark$ & $\checkmark$ & $\checkmark$ \\
\hline 17 & - & $\checkmark$ & $\checkmark$ & $\checkmark$ & - & - & - & - & - & - & - & - & - & - & - & - & - & - & - \\
\hline 18 & $\checkmark$ & - & $\checkmark$ & - & - & - & - & - & - & - & - & - & - & - & - & - & - & - & - \\
\hline 19 & - & - & $\checkmark$ & - & $\checkmark$ & - & $\checkmark$ & $\checkmark$ & $\checkmark$ & - & - & - & - & - & - & - & - & $\checkmark$ & $\checkmark$ \\
\hline 20 & - & - & - & - & - & - & - & - & - & - & - & - & - & - & - & - & $\checkmark$ & - & - \\
\hline 21 & $\checkmark$ & - & - & - & $\checkmark$ & - & - & - & - & - & - & - & - & - & $\checkmark$ & - & $\checkmark$ & $\checkmark$ & $\checkmark$ \\
\hline 22 & $\checkmark$ & $\checkmark$ & $\checkmark$ & - & - & - & - & - & - & - & - & - & - & - & - & - & - & - & - \\
\hline 23 & $\checkmark$ & $\checkmark$ & $\checkmark$ & - & - & - & - & - & - & - & - & - & - & - & - & - & - & - & $\checkmark$ \\
\hline 24 & $\checkmark$ & - & $\checkmark$ & - & - & - & - & - & - & $\checkmark$ & - & $\checkmark$ & - & $\checkmark$ & - & - & - & - & - \\
\hline 25 & - & $\checkmark$ & $\checkmark$ & - & - & $\checkmark$ & - & - & $\checkmark$ & - & - & - & - & - & - & - & - & $\checkmark$ & - \\
\hline 26 & $\checkmark$ & - & $\checkmark$ & - & $\checkmark$ & $\checkmark$ & $\checkmark$ & $\checkmark$ & $\checkmark$ & - & - & - & - & - & - & - & $\checkmark$ & $\checkmark$ & $\checkmark$ \\
\hline 27 & - & - & $\checkmark$ & - & $\checkmark$ & - & - & $\checkmark$ & - & - & - & - & - & - & - & - & - & $\checkmark$ & - \\
\hline 28 & - & $\checkmark$ & - & - & - & - & - & - & - & - & - & - & - & - & $\checkmark$ & - & - & - & - \\
\hline 29 & $\checkmark$ & $\checkmark$ & $\checkmark$ & - & - & - & - & - & $\checkmark$ & - & - & - & - & - & - & - & $\checkmark$ & $\checkmark$ & - \\
\hline 30 & $\checkmark$ & - & $\checkmark$ & - & - & - & - & - & - & - & - & - & - & - & - & - & - & - & $\checkmark$ \\
\hline 31 & $\checkmark$ & - & $\checkmark$ & $\checkmark$ & $\checkmark$ & - & - & $\checkmark$ & - & $\checkmark$ & - & - & - & - & - & - & - & - & - \\
\hline 32 & $\checkmark$ & - & $\checkmark$ & - & $\checkmark$ & - & - & - & - & - & - & - & - & - & - & - & - & - & $\checkmark$ \\
\hline 33 & $\checkmark$ & $\checkmark$ & $\checkmark$ & - & $\checkmark$ & - & - & - & $\checkmark$ & - & - & - & - & - & $\checkmark$ & - & - & - & - \\
\hline 34 & $\checkmark$ & - & $\checkmark$ & - & $\checkmark$ & - & - & $\checkmark$ & $\checkmark$ & $\checkmark$ & - & - & - & - & $\checkmark$ & - & - & $\checkmark$ & - \\
\hline 35 & - & $\checkmark$ & $\checkmark$ & - & - & $\checkmark$ & - & $\checkmark$ & $\checkmark$ & - & - & - & - & - & - & - & - & $\checkmark$ & - \\
\hline 36 & $\checkmark$ & $\checkmark$ & $\checkmark$ & $\checkmark$ & - & - & - & - & - & - & - & - & - & - & $\checkmark$ & $\checkmark$ & - & - & - \\
\hline 37 & - & $\checkmark$ & $\checkmark$ & - & $\checkmark$ & - & - & - & - & - & - & - & - & - & - & - & - & - & - \\
\hline 38 & - & $\checkmark$ & $\checkmark$ & - & $\checkmark$ & - & - & - & - & - & - & - & - & - & - & - & - & - & $\checkmark$ \\
\hline \begin{tabular}{|l|}
9 \\
\end{tabular} & $\checkmark$ & - & $\checkmark$ & - & $\checkmark$ & $\checkmark$ & $\checkmark$ & $\checkmark$ & $\checkmark$ & $\checkmark$ & - & - & $\checkmark$ & - & $\checkmark$ & $\checkmark$ & $\checkmark$ & $\checkmark$ & $\checkmark$ \\
\hline 40 & - & $\checkmark$ & $\checkmark$ & - & - & - & $\checkmark$ & $\checkmark$ & $\checkmark$ & - & - & - & - & - & - & - & - & $\checkmark$ & $\checkmark$ \\
\hline 41 & - & - & $\checkmark$ & - & - & - & - & - & - & - & - & - & - & - & $\checkmark$ & $\checkmark$ & - & $\checkmark$ & - \\
\hline 42 & $\checkmark$ & $\checkmark$ & $\checkmark$ & - & $\checkmark$ & - & - & $\checkmark$ & - & - & - & - & - & - & - & - & $\checkmark$ & $\checkmark$ & - \\
\hline 43 & - & $\checkmark$ & - & $\checkmark$ & - & & - & - & - & - & - & - & - & - & - & - & - & - & - \\
\hline 44 & $\checkmark$ & $\checkmark$ & $\checkmark$ & - & $\checkmark$ & - & - & $\checkmark$ & $\checkmark$ & - & - & - & - & - & - & - & $\checkmark$ & $\checkmark$ & - \\
\hline 45 & - & $\checkmark$ & $\checkmark$ & - & - & - & - & $\checkmark$ & - & - & - & - & - & - & - & - & - & - & - \\
\hline 46 & - & $\checkmark$ & - & - & - & - & $\checkmark$ & $\checkmark$ & $\checkmark$ & - & - & - & - & - & $\checkmark$ & $\checkmark$ & $\checkmark$ & $\checkmark$ & - \\
\hline 47 & $\checkmark$ & $\checkmark$ & $\checkmark$ & - & $\checkmark$ & - & - & $\checkmark$ & - & - & - & - & - & - & $\checkmark$ & $\checkmark$ & $\checkmark$ & $\checkmark$ & - \\
\hline 48 & $\checkmark$ & - & $\checkmark$ & - & $\checkmark$ & - & - & $\checkmark$ & $\checkmark$ & - & - & - & - & - & $\checkmark$ & $\checkmark$ & $\checkmark$ & $\checkmark$ & $\checkmark$ \\
\hline 49 & - & - & $\checkmark$ & - & - & - & $\checkmark$ & $\checkmark$ & - & - & - & - & - & - & $\checkmark$ & - & $\checkmark$ & $\checkmark$ & - \\
\hline 50 & - & $\checkmark$ & $\checkmark$ & - & - & - & - & $\checkmark$ & - & - & - & - & - & - & $\checkmark$ & $\checkmark$ & - & $\checkmark$ & $\checkmark$ \\
\hline
\end{tabular}


Table 15: continued from previous page

\begin{tabular}{|c|c|c|c|c|c|c|c|c|c|c|c|c|c|c|c|c|c|c|c|}
\hline \multirow[b]{2}{*}{ 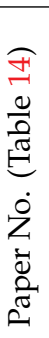 } & \multicolumn{8}{|c|}{ Cost Parameters } & \multicolumn{6}{|c|}{ Financial Parameters } & \multicolumn{2}{|c|}{ Prod./Proc. } & \multirow[b]{2}{*}{ 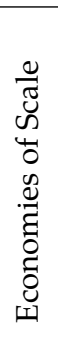 } & \multirow[b]{2}{*}{ 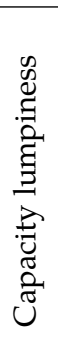 } & \multirow[b]{2}{*}{ 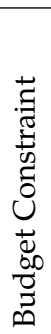 } \\
\hline & 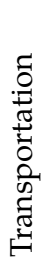 & 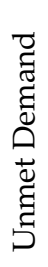 & 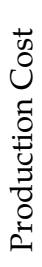 & $\begin{array}{l}\overrightarrow{0} \\
0 \\
0 \\
\vdots \\
0 \\
0 \\
0 \\
0\end{array}$ & 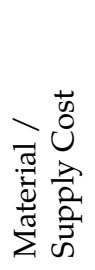 & 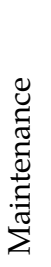 & 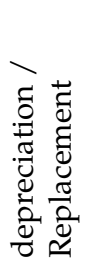 & 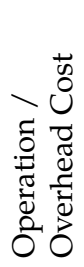 & 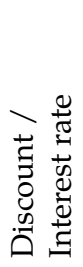 & త & $\frac{2}{2}$ & $\underset{\$}{\mathbb{S}}$ & 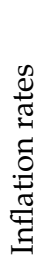 & 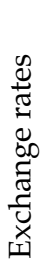 & 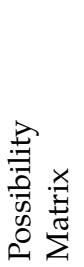 & 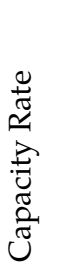 & & & \\
\hline 51 & - & - & $\checkmark$ & - & - & - & - & $\checkmark$ & - & - & - & - & - & - & $\checkmark$ & $\checkmark$ & - & $\checkmark$ & $\checkmark$ \\
\hline 52 & $\checkmark$ & - & $\checkmark$ & - & $\checkmark$ & - & - & $\checkmark$ & - & - & - & - & - & - & $\checkmark$ & - & - & - & - \\
\hline 53 & - & - & $\checkmark$ & $\checkmark$ & - & - & - & $\checkmark$ & - & - & - & - & - & - & $\checkmark$ & $\checkmark$ & - & $\checkmark$ & - \\
\hline 54 & - & - & $\checkmark$ & - & - & - & $\checkmark$ & $\checkmark$ & - & - & - & - & - & - & $\checkmark$ & $\checkmark$ & $\checkmark$ & $\checkmark$ & - \\
\hline 55 & $\checkmark$ & $\checkmark$ & $\checkmark$ & - & $\checkmark$ & - & - & $\checkmark$ & - & - & $\checkmark$ & - & - & - & - & - & - & $\checkmark$ & - \\
\hline 56 & $\checkmark$ & - & $\checkmark$ & - & - & - & - & - & - & - & - & - & - & - & $\checkmark$ & $\checkmark$ & - & - & $\checkmark$ \\
\hline 57 & - & - & - & - & $\bar{\checkmark}$ & - & - & - & - & - & - & - & - & - & - & - & - & - & - \\
\hline 58 & $\checkmark$ & - & - & - & - & - & - & - & - & - & - & - & - & - & $\checkmark$ & $\checkmark$ & $\checkmark$ & - & - \\
\hline 59 & - & $\checkmark$ & $\checkmark$ & $\checkmark$ & - & - & - & $\checkmark$ & - & - & - & - & - & - & $\checkmark$ & $\checkmark$ & - & $\checkmark$ & - \\
\hline 60 & $\checkmark$ & - & - & - & $\checkmark$ & $\checkmark$ & - & - & $\checkmark$ & - & - & - & $\checkmark$ & - & - & - & $\checkmark$ & $\checkmark$ & $\checkmark$ \\
\hline 61 & $\checkmark$ & $\checkmark$ & $\checkmark$ & - & $\bar{\checkmark}$ & - & - & - & - & - & - & - & - & - & - & - & - & - & - \\
\hline 62 & - & $\checkmark$ & $\checkmark$ & - & - & - & - & - & $\checkmark$ & - & - & - & - & - & $\checkmark$ & - & - & - & - \\
\hline 63 & $\checkmark$ & - & $\checkmark$ & - & $\checkmark$ & - & - & $\checkmark$ & - & - & - & - & - & - & $\checkmark$ & $\checkmark$ & - & - & - \\
\hline 64 & - & $\checkmark$ & $\checkmark$ & - & - & $\checkmark$ & $\checkmark$ & $\checkmark$ & - & - & - & - & - & - & - & - & - & - & $\checkmark$ \\
\hline 65 & - & $\checkmark$ & $\checkmark$ & - & - & - & - & - & - & - & - & - & - & - & $\checkmark$ & - & - & - & - \\
\hline 66 & - & $\checkmark$ & $\checkmark$ & - & - & - & - & - & - & - & - & - & - & - & $\checkmark$ & $\checkmark$ & - & $\checkmark$ & - \\
\hline 67 & $\checkmark$ & - & $\checkmark$ & - & $\checkmark$ & - & - & $\checkmark$ & - & $\checkmark$ & - & - & - & - & $\checkmark$ & - & - & - & - \\
\hline 68 & $\checkmark$ & $\checkmark$ & $\checkmark$ & - & $\checkmark$ & - & - & $\checkmark$ & - & - & - & - & - & - & - & - & $\checkmark$ & - & $\checkmark$ \\
\hline 69 & - & $\checkmark$ & - & - & - & - & - & $\checkmark$ & - & - & - & - & - & - & - & - & - & $\checkmark$ & $\checkmark$ \\
\hline
\end{tabular}

\section{References}

Ackermann, F., Franco, L. A., Rouwette, E., and White, L. (2014). Special issue on problem structuring research and practice.

Afrouzy, Z. A., Nasseri, S. H., Mahdavi, I., and Paydar, M. M. (2016). A fuzzy stochastic multi-objective optimization model to configure a supply chain considering new product development. Applied Mathematical Modelling, 40(17-18):7545-7570.

Aghezzaf, E., Sitompul, C., and Najid, N. M. (2010). Models for robust tactical planning in multi-stage production systems with uncertain demands. Computers $\mathcal{E}$ Operations Research, 37:880-889.

Ahmed, S., King, A. J., and Parija, G. (2003). A multi-stage stochastic integer programming approach for capacity expansion under uncertainty. Journal of Global Optimization, 26:3-24.

Ahmed, S. and Sahinidis, N. V. (2008). Selection, acquisition, and allocation of manufacturing technology in a multi-period environment. European Journal of Operational Research, 189:807821.

Ambrosi, M. (2010). Development of Strategic Planning Model for Automotive Supplier.

Azaron, A., Brown, K. N., Tarim, S. A., and Modarres, M. (2008). A multi-objective stochastic programming approach for supply chain design considering risk. International Journal of Production Economics, 116:129-138.

Barahona, F., Bermon, S., Gunluk, O., and Hood, S. (2005). Robust capacity planning in semiconductor manufacturing. Naval Research Logistics, 52:459-468.

Bawden, T. and Lewis, L. (2010). Toyota may close uk production line but pledges to retain staff - times online. 2010. 
Bekker, H. (2010). Best-selling car manufacturers in britain (2009): List of the top 30 new passenger vehicle registrations in the uk. 2010.

Bhutta, K. S., Huq, F., Frazier, G., and Mohamed, Z. (2003). An integrated location, production, distribution and investment model for a multinational corporation. International Journal of Production Economics, 86:201-216.

Bihlmaier, R., Koberstein, A., and Obst, R. (2010). Modeling and optimizing of strategic and tactical production planning in the automotive industry under uncertainty. OR Spectrum, 31:311-336.

Ceryan, O. and Koren, Y. (2009). Manufacturing capacity planning strategies. CIRP Annals Manufacturing Technology, 58:403-406.

Chakravarty, A. K. (2005). Global plant capacity and product allocation with pricing decisions. European Journal of Operational Research, 165:157-181.

Chandra, C., Everson, M., and Grabis, J. (2005). Evaluation of enterprise-level benefits of manufacturing flexibility. Omega, 33:17-31.

Chauhan, S. S., Nagi, R., and Proth, J. (2004). Strategic capacity planning in supply chain design for a new market opportunity. International Journal of Production Research, 42:2197-2206.

Chen, G., Daskin, M. S., Shen, Z. J. M., and Uryasev, S. (2006). The Îś-reliable mean-excess regret model for stochastic facility location modeling. Naval Research Logistics, 53:617-626.

Chen, T. 1., Lin, J. T., and Wu, C. H. (2014). Coordinated capacity planning in two-stage thinfilm-transistor liquid-crystal-display (TFT-LCD) production networks. Omega, 42:141-156.

Chen, T. L. and Lu, H. C. (2012). Stochastic multi-site capacity planning of TFT-LCD manufacturing using expected shadow-price based decomposition. Applied Mathematical Modelling, 36:5901-5919.

Chen, Y. Y. and Fan, H. Y. (2015). An application of stochastic programming in solving capacity allocation and migration planning problem under uncertainty. Mathematical Problems in Engineering, 2015:1-16.

Chen, Z., Li, S., and Tirupati, D. (2002). A scenario-based stochastic programming approach for technology and capacity planning. Computers $\mathcal{E}$ Operations Research, 29:781-806.

Chien, C., Wu, C., and Chiang, Y. (2012). Coordinated capacity migration and expansion planning for semiconductor manufacturing under demand uncertainties. International Journal of Production Economics, 135:860-869.

Chien, C.-F., Dou, R., and Fu, W. (2018). Strategic capacity planning for smart production: Decision modeling under demand uncertainty. Applied Soft Computing, 68:900-909.

Claro, J. and Pinho de Sousa, J. (2012). A multiobjective metaheuristic for a mean-risk multistage capacity investment problem with process flexibility. Computers $\mathcal{E}$ Operations Research, 39:838849 .

Colvin, M. and Maravelias, C. T. (2009). Scheduling of testing tasks and resource planning in new product development using stochastic programming. Computers $\mathcal{E}$ Chemical Engineering, 33:964-976.

Costea, A. (2010). Toyota reports first half fiscal year 2011 financial results. 2010.

Dal-Mas, M., Giarola, S., Zamboni, A., and Bezzo, F. (2011). Strategic design and investment capacity planning of the ethanol supply chain under price uncertainty. Biomass and Bioenergy, 35:2059-2071. 
Durksen, D. and Dangelmaier, W. (2010). A model of a system for hierarchical planning of structure and dimension of internal global production networks. System Sciences (HICSS), 2010(43):1-10.

Eppen, G. D., Martin, R. K., and Schrage, L. (1989). A scenario approach to capacity planning. A Scenario Approach to Capacity Planning, 37:517-527.

Erbis, S., Kamarthi, S., Cullinane, T. P., and Isaacs, J. A. (2014). Multistage stochastic programming (msp) model for carbon nanotube production capacity expansion planning. $C S$ Sustainable Chemistry \& Engineering, 2:1633-1641.

Farahani, R. Z., SteadieSeifi, M., and Asgari, N. (2010). Multiple criteria facility location problems: A survey. Applied Mathematical Modelling, 34:1689-1709.

Ferdows, K. (1997). Making the most of foreign factories. Harvard Business Review Article, 75:73-88.

Fleischmann, B., Ferber, S., and Henrich, P. (2006). Strategic planning of BMW's global production network. Interfaces, 36(3):194-208.

Francas, D., Kremer, M., Minner, S., and Friese, M. (2009). Strategic process flexibility under lifecycle demand. International Journal of Production Economics, 121:427-440.

Frausto-Hernandez, S., Rico-Ramirez, V., and Grossmann, I. E. (2010). Strategic capacity allocation under uncertainty by using a two-stage stochastic decomposition algorithm with incumbent solutions. Industrial E Engineering Chemistry Research, 49:2812-2821.

Garcia-Herreros, P., Zhang, L., Misra, P., Arslan, E., Mehta, S., and Grossmann, I. E. (2016). Mixed-integer bilevel optimization for capacity planning with rational markets. Computers $\mathcal{E}$ Chemical Engineering, 86:33-47.

Gatica, G., Papageorgiou, L. G., and Shah, N. (2003). Capacity planning under uncertainty for the pharmaceutical industry. Chemical Engineering Research and Design, 81:665-678.

Geng, N., Jiang, Z., and Chen, F. (2009). Stochastic programming based capacity planning for semiconductor wafer fab with uncertain demand and capacity. European Journal of Operational Research, 198:899-908.

Goel, V. and Grossmann, I. E. (2004). A stochastic programming approach to planning of offshore gas field developments under uncertainty in reserves. Computers $\mathcal{E}$ Chemical Engineering, 28:1409-1429.

Graves, S. C. (2011). Uncertainty and production planning. In K. Kempf, G., Keskinocak, P., and Uzsoy, R., editors, planning production and inventories in the extended enterprise, pages 83-102. New York, Springer.

Gupta, M. and Mohanty, B. K. (2015). Multi-stage multi-objective production planning using linguistic and numeric data-a fuzzy integer programming model. Computers $\mathcal{E}$ Industrial Engineering, 87:454-464.

Hamad, R. and Fares Gualda, N. D. (2008). Model for facilities or vendors location in a global scale considering several echelons in the chain. Networks and Spatial Economics, 8:297-307.

Hammami, R., Frein, Y., and Hadj-Alouane, A. B. (2008). Supply chain design in the delocalization context: Relevant features and new modeling tendencies. International Journal of Production Economics, 113:641-656.

Hammami, R., Frein, Y., and Hadj-Alouane, A. B. (2009). A strategic-tactical model for the supply chain design in the delocalization context: Mathematical formulation and a case study. International Journal of Production Economics, 122:351-365. 
Hamta, N., Shirazi, A., M., F. G., T., S. M., and Behdad, S. (2015). Supply chain network optimization considering assembly line balancing and demand uncertainty. International Journal of Production Research, 53:2970-2994.

Hvolby, H. and Steger-Jensen, K. (2010). Technical and industrial issues of advanced planning and scheduling (APS) systems. Computers in Industry, 61:845-851.

Inman, R. R. and Gonsalvez, D. J. A. (2001). A mass production product-to-plant allocation problem. Computers $\mathcal{E}$ Industrial Engineering, 39:255-271.

Julka, N. (2008). A Screening Model to Explore Planning Decisions in Automotive Manufacturing Systems under Demand Uncertainty.

Julka, N., Baines, T., Tjahjono, B., Lendermann, P., and Vitanov, V. (2007). A review of multifactor capacity expansion models for manufacturing plants: Searching for a holistic decision aid. International Journal of Production Economics, 106:607-621.

Katayama, N., Yurimoto, S., and Yun, S. (2007). A Product-to-Plant Allocation Problem in Logistics Network Design.

Kauder, S. and Meyr, H. (2009). Strategic network planning for an international automotive manufacturer. OR Spectrum, 31:507-532.

Kaya, O., Bagci, F., and Turkay, M. (2014). Planning of capacity, production and inventory decisions in a generic reverse supply chain under uncertain demand and returns. International Journal of Production Research, 52:270-282.

Lea, R. (2010). Toyota ponders temporary closure of british factory as sales collapse worldwide - times online. 2010.

Lee, C. Y. and Johnson, A. L. (2014). Proactive data envelopment analysis: effective production and capacity expansion in stochastic environments. European Journal of Operational Research, 232:537-548.

Lin, J. T., Chen, T. L., and Chu, H. C. (2014). A stochastic dynamic programming approach for multi-site capacity planning in TFT-LCD manufacturing under demand uncertainty. International Journal of Production Economics, 148:21-36.

Lin, J. T., Wu, C., Chen, T., and Shih, S. (2010). A stochastic programming model for strategic capacity planning in thin film transistor-liquid crystal display TFT-LCD industry. Computers E Operations Research, 38:992-1007.

Liu, H., Zhao, Q., Huang, N., and Zhao, X. (2015). Production line capacity planning concerning uncertain demands for a class of manufacturing systems with multiple products. IEEE/CAA Journal of Automatica Sinica, 2(2):217-225.

Liu, Y., Sioshansi, R., and Conejo, A. J. (2018). Multistage stochastic investment planning with multiscale representation of uncertainties and decisions. IEEE Transactions on Power Systems, 33(1):781-791.

Lusa, A. and Pastor, R. (2011). Planning working time accounts under demand uncertainty. Computers \& Operations Research, 38:517-524.

Luss, H. (1982). Operations research and capacity expansion problems: A survey. Operations research, 30:907-947.

MacCormack, A. D., Newmann, L. J. I., and Rosenfield, D. B. (1994). The new dynamics of global manufacturing site location. MIT Sloan Management Review, 35:69-84. 
Matta, A., Semeraro, Q., and Tolio, T. (2005). A framework for long term capacity decisions in amss. In Matta and Semeraro, Q., editors, Design of advanced manufacturing systems: models for capacity planning in Advanced Manufacturing Systems, pages 1-36. Springer, Netherlands.

Medina-González, S., Pozo, C., Corsano, G., Guillén-Gósalbez, G., and Espuña, A. (2017). Using pareto filters to support risk management in optimization under uncertainty: Application to the strategic planning of chemical supply chains. Computers \& Chemical Engineering, 98:236255.

Melo, M. T., Nickel, S., and Saldanha da Gama, F. (2006). Dynamic multi-commodity capacitated facility location: a mathematical modeling framework for strategic supply chain planning. Computers E Operations Research, 33:181-208.

Melo, M. T., Nickel, S., and Saldanha-da Gama, F. (2009). Facility location and supply chain management - a review. European Journal of Operational Research, 196:401-412.

Mula, J., Poler, R., García-Sabater, J. P., and Lario, F. C. (2006). Models for production planning under uncertainty: A review. International Journal of Production Economics, 103:271-285.

Nagar, L. and Jain, K. (2008). Supply chain planning using multi-stage stochastic programming. Supply Chain Management: An International Journal, 13:251-256.

Naraharisetti, P. K. and Karimi, I. A. (2010). Supply chain redesign and new process introduction in multipurpose plants. Chemical Engineering Science, 65:2596-2607.

Nickel, S., Saldanha-da Gama, F., and Ziegler, H. (2012). A multi-stage stochastic supply network design problem with financial decisions and risk management. Omega, 40:511-524.

Nodem, D., I., F., Kenne, J. P., and Gharbi, A. (2008). Joint optimization of capacity expansion, production and maintenance in stochastic manufacturing systems. Applied Mathematical Science, 2:195-212.

Olhager, J., Rudberg, M., and Wikner, J. (2001). Long-term capacity management: Linking the perspectives from manufacturing strategy and sales and operations planning. International Journal of Production Economics, 69:215-225.

Papageorgiou, A. G., Rotstein, G. E., and Shah, N. (2001). Strategic supply chain optimization for the pharmaceutical industries. Industrial \& Engineering Chemistry Research, 40:275-286.

Peidro, D., Mula, J., Poler, R., and Verdegay, J. (2009). Fuzzy optimization for supply chain planning under supply, demand and process uncertainties. Fuzzy Sets and Systems, 160:26402657.

Pidd, M. (2003). Tools for Thinking: Modelling in Management Science. (2nd ed.). UK, John Wiley \& Sons.

Pimentel, B. S., Mateus, G. R., and Almeida, F. A. (2013). Stochastic capacity planning and dynamic network design. International Journal of Production Economics, 145:139-149.

Ruddick, G. (2010). Toyota 'sincerely grateful' as it returns to profit despite recall crisis. 2010.

Sabet, E. (2012). A strategic decision making model on global capacity management for the manufacturing industry under market uncertainty. PhD thesis, Nottingham Trent University.

Shiina, T., Takaichi, T., Li, Y., Morito, S., and Imaizumi, J. (2018). Multistage stochastic programming model and solution algorithm for the capacity expansion of railway network. Journal of Advanced Mechanical Design, Systems, and Manufacturing, 12(3):JAMDSM0077-JAMDSM0077.

Snyder, L. V. (2006). Facility location under uncertainty: A review. IIE Transportations, 38:537554. 
Snyder, L. V., Daskin, M. S., and Teo, C. (2007). The stochastic location model with risk pooling. European Journal of Operational Research, 179:1221-1238.

Sterman, J. D. (2000). Business Dynamics: Systems Thinking and Modeling for a Complex World. (1st ed.). Irwin, McGraw-Hill.

Syam, S. S. (2000). Multiperiod capacity expansion in globally dispersed region. Decision Science, 31:173-195.

Tarhan, B. and Grossmann, I. E. (2008). A multistage stochastic programming approach with strategies for uncertainty reduction in the synthesis of process networks with uncertain yields. Computers \& Chemical Engineering, 32:766-788.

Telegraph, T. (2010). Toyota to halt production at british plants. 2010.

Tenhiälä, A. (2011). Contingency theory of capacity planning: The link between process types and planning methods. Journal of Operations Management, 29:65-77.

Verma, A., Shukla, N., Tyagi, S., and Mishra, N. (2014). Stochastic modelling and optimisation of multi-plant capacity planning problem. International Journal of Intelligent Engineering Informatics, 2:139-165.

Verter, V. and Dasci, A. (2002). The plant location and flexible technology acquisition problem. European Journal of Operational Research, 136:366-382.

Wagner, M. R., Bhadury, J., and Peng, S. (2009). Risk management in uncapacitated facility location models with random demands. Computers $\mathcal{E}$ Operations Research, 36:1002-1011.

Wang, K.-J. and Nguyen, P. H. (2017). Capacity planning with technology replacement by stochastic dynamic programming. European Journal of Operational Research, 260(2):739-750.

Wang, K.-J., Nguyen, P. H., Wu, S.-Y., and Xue, J. (2018). Technology portfolio adoption considering capacity planning under demand and technology uncertainty. Journal of Manufacturing Systems, 47:1-11.

Wu, C. and Chuang, Y. (2010). An innovative approach for strategic capacity portfolio planning under uncertainties. European Journal of Operational Research, 207:1002-1013.

Yang, F., Gao, K., Simon, I. W., Zhu, Y., and Su, R. (2018). Decomposition methods for manufacturing system scheduling: a survey. IEEE/CAA Journal of Automatica Sinica, 5(2):389-400.

You, F., Wassick, J. M., and Grossmann, I. E. (2009). Risk management for a global supply chain planning under uncertainty: Models and algorithms. AIChE Journal, 55:931-946.

Yu, H. and Solvang, W. D. (2018). Incorporating flexible capacity in the planning of a multiproduct multi-echelon sustainable reverse logistics network under uncertainty. Journal of Cleaner Production, 198:285-303.

Zhang, R. (2007). Research on capacity planning under stochastic production and uncertain demand. Systems Engineering - Theory E Practice, 27:51-59.

Zhou, R.-J. and Li, L.-J. (2018). Joint capacity planning and distribution network optimization of coal supply chains under uncertainty. AIChE Journal, 64(4):1246-1261. 